\title{
Notch signaling determines cell-fate specification of the two main types of vomeronasal neurons of rodents.
}

Raghu Ram Katreddi ${ }^{1,2,3}$, Ed Zandro M.Taroc ${ }^{1,2,3}$, Sawyer M Hicks ${ }^{1,2}$, Jennifer M Lin ${ }^{1,2,3}$, Shuting Liü ${ }^{4}$ Mengqing Xiang ${ }^{4}$ and Paolo E. Forni ${ }^{1,2,3 *}$

1) Department of Biological Sciences, 2) The RNA Institute and 3) The Center for Neuroscience Research, University at Albany, State University of New York, Albany, NY, United States.

4) State Key Laboratory of Ophthalmology, Zhongshan Ophthalmic Center, Sun Yat-sen University, Guangzhou 510060, China

* corresponding author: pforni@albany.edu

\section{Graphical Abstract}

\begin{tabular}{|c|c|c|c|}
\hline $\begin{array}{l}\text { Notch signaling triggers } \\
\text { the apical-basal VSNs' dichotomy }\end{array}$ & $\begin{array}{l}\text { Notch Ablation at Ascl1 stage } \\
\text { makes apical VSNs the default program }\end{array}$ & $\begin{array}{l}\text { Ectopic NICD at Ascl1 stage } \\
\text { mosity makes sustentacular cells }\end{array}$ & $\begin{array}{l}\text { Ectopic NICD at Neurog } 1 \text { stage } \\
\text { makes basal VSNs the default program }\end{array}$ \\
\hline Wild type animals & 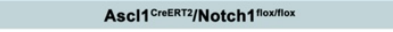 & 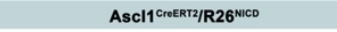 & Neurog 1 CreERT/R26 $6^{\text {NCD }}$ \\
\hline 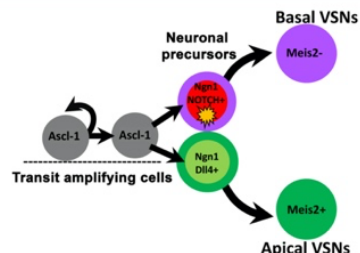 & $A D$ & Sustentacular Cells & Meiz2. \\
\hline
\end{tabular}

\section{Abstract:}

The ability of terrestrial vertebrates to find food, mating partners and to avoid predators heavily relies on the detection of chemosensory information from the environment. The olfactory system of most vertebrate species comprises two distinct chemosensory systems usually referred to as the main and the accessory olfactory system. Olfactory sensory neurons of the main olfactory epithelium detect and transmit odor information to main olfactory bulb (MOB), while the chemosensory neurons of the vomeronasal organ detect semiochemicals responsible for social and sexual behaviors and transmit information to the accessory olfactory bulb (AOB). The vomeronasal sensory epithelium (VNE) of most mammalian species contains uniform vomeronasal (VN) system with vomeronasal sensory neurons (VSNs) expressing vomeronasal receptors of the V1R family. However, rodents and some marsupials have developed a more complex binary VN system, where VNO containing a second main type of VSNs expressing vomeronasal 
receptors of the V2R family is identified. In mice, V1R and V2R VSNs form from a common pool of progenitors but have distinct differentiation programs. As they mature, they segregate in different regions of the VNE and connect with different parts of the AOB. How these two main types of VSNs are formed has never been addressed. In this study, using single cell RNA sequencing data, we identified differential expression of Notch1 receptor and DII4 ligand among the neuronal precursors at the VSN dichotomy. We further demonstrated with loss of function (LOF) and gain of function (GOF) studies that Dll4-Notch1 signaling plays a crucial role in triggering the binary dichotomy between the two main types of VSNs in mice.

Keywords: Single cell sequencing, Vomeronasal Organ, Notch signaling, DII4, neuronal dichotomy, mouse, neuronal differentiation

Introduction: Neural stem/progenitor cells can give rise to multiple neuronal cell types that differ in gene expression, functions, and neuronal connectivity. Investigating the molecular mechanisms that establish different neuronal cell-fates is crucial to understand how neuronal systems evolve, form and, to identify molecular mechanisms underlying neurodevelopmental disorders [1-3].

The vomeronasal organ (VNO) is a specialized chemosensory organ that, in many vertebrate species, is located at the base of the nasal cavity [4]. The VNO is responsible for the detection of semiochemicals, molecules that can trigger stereotypical mating/sex behaviors, parental behaviors, and predator avoidance [5-8]. Most of the vertebrates having a functional VNO, have neurons expressing vomeronasal receptor genes of the V1R family [9]. However, the vomeronasal sensory epithelium (VNE) of mice, rats and opossum consists of two main types of vomeronasal neurons [10]. VSNs that express Gai2 G protein subunit, receptors of the V1R family and the transcription factor Meis2 are fairly conserved population across many vertebrate species [11]. The second population that has been reported in rodents and few other animal species is formed by the VSNs that express the Gao $G$ protein subunit, receptors of the V2R family and the transcription factor tfap2e (AP-2 $\varepsilon$ ) [11-14]. In mice, the V1R expressing neurons are mostly distributed in the apical territories of VNE and for this reason they are often referred to as apical 
VSNs. Conversely, the V2R expressing neurons that, for the most part, are located in basal regions of the VNE and around the vasculature, are called basal VSNs [15]. Both apical and basal VSNs detect distinct types of ligands, connect to different areas of the accessory olfactory bulb and control distinct behaviors [16-19].

In mice, the VSN neurogenesis starts during embryonic development around E11.5 and continues, throughout the life, starting from a limited number of progenitors in the marginal zones of the VNO [15, 20-23]. How the cell fate of apical and basal VSN types is established has not been fully understood. A pivotal study by Enomoto and coworkers previously identified that the transcription factor Bcl11b plays a key role in controlling the correct establishment of apical and basal VSNs in the developing VNO [11]. However, what extrinsic signaling pathways control the expression of Bcl11b in the VNO has not been investigated until now. Moreover, the same study identified, the transcription factor AP- $2 \varepsilon$ as a potential target of $\mathrm{Bcl} 11 \mathrm{~b}$ and suggested a role for AP- $2 \varepsilon$ in controlling basal neuron differentiation. In a follow-up study, we proposed that the AP$2 \varepsilon$ is not responsible for initiating the apical vs basal identity bifurcation but is crucial for the expression of several genes defining, and maintaining, the basal VSN's molecular identity [14]. In fact, we observed that after AP-2 $\varepsilon$ loss of function, VSNs with basal identity lose the expression of basal genes (Gao and V2Rs) while they switch to expressing apical genes such as Gai2 and V1Rs [14].

In this study we adopted a single cell sequencing strategy to investigate the mechanisms involved in cell fate specification and differentiation of apical and basal VSNs. By following the transcriptomic profile of postnatal stem cells/progenitors and immature VSNs, we identified differential expression of Notch-1 receptor and DII4 ligand across Neurog1/Neurod1 positive VSNs' precursors. Following this hint, we adopted both loss of function (LOF) and gain of function (GOF) in-vivo strategies to test the role of this signaling pathway in establishing the VSNs' binary differentiation. LOF and GOF strategies differentially affected the switch of apical vs basal VSN cell fate with Notch signaling LOF directing cells towards apical and GOF towards basal VSN fate. Our data on the VNO offer a new example of how Notch-mediated cell fate choice is a conserved strategy for establishing binary cellular diversification and increasing the neuronal repertoire in developing neuroepithelia. 


\section{RESULTS}

\section{Single cell profiling of the whole adult VNO identifies VSN dichotomy.}

In mice, adult

neurogenesis is

seen throughout

the life at marginal

zones of the

vomeronasal

epithelium [23, 24].

To identify multiple

cell types like stem

cells, VSNs and

non-neuronal cell

types,

we

performed single

cell RNA

sequencing (sc-

RNA seq) from

dissociated whole

VNO. We used

Seurat, R package,

to filter out low

quality cells and

perform clustering

and analyze sc-

RNA-seq data [25].

A total of 10,582

single-cell

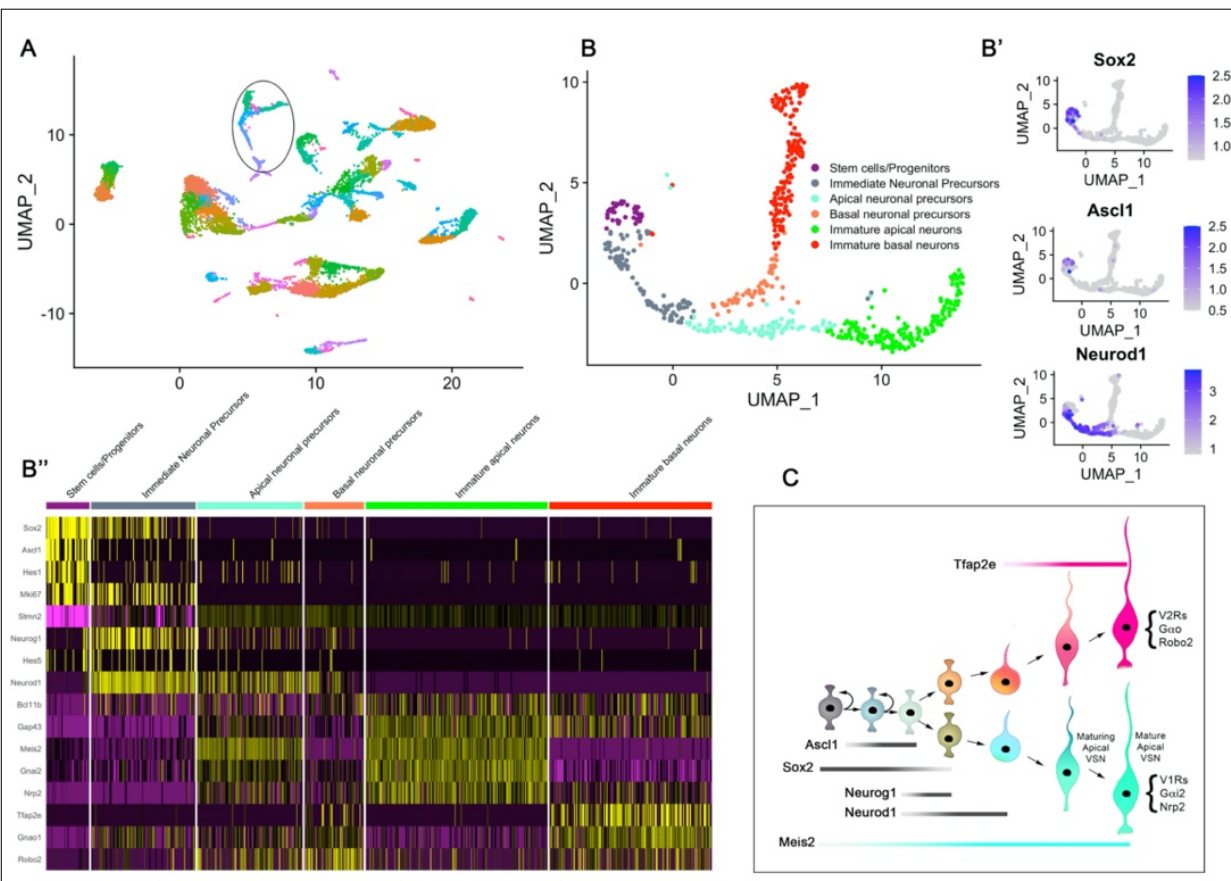

D

D'
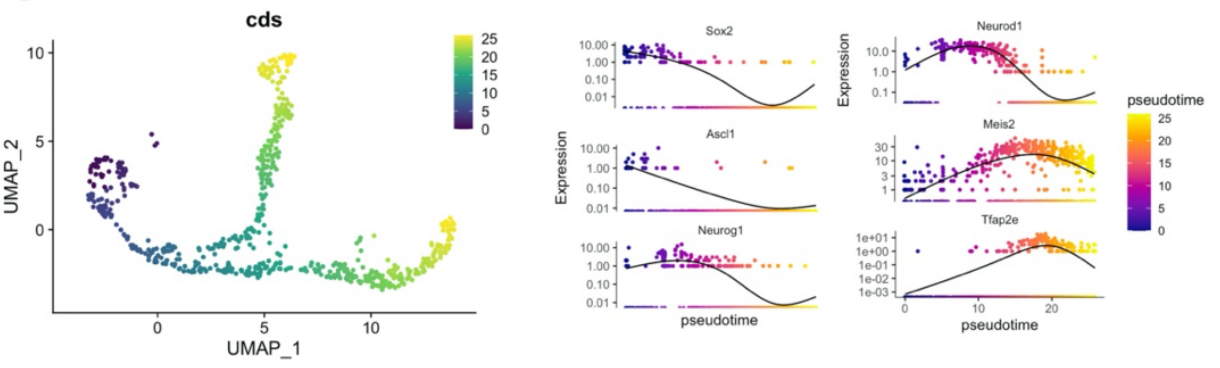

Figure1: sc-RNA-seq identified adult neurogenesis and VSN apical-basal dichotomy in the mouse VNO. A) UMAP dimensional reduction plot of Seurat object1 shows neuronal and nonneuronal cell clusters of VNO. Each color corresponds to a cluster of cells that have similar transcriptomic profile. Clusters that are circled consists of neuronal progenitors, precursors and immature VSN apical and basal populations. B) Seurat object2 generated from highlighted clusters of Seurat objet 1 (encircled) distinguishes VSN neuronal dichotomy and identifies cell clusters specific to neuronal progenitors, immediate neuronal precursors, apical and, basal neuronal precursors and apical and, basal immature VSNs. B') Feature plots of Sox2, Ascl1 and Neurod1 in Seurat object2. B') Heatmap of the known genes that are specific to each cluster. C) Summary cartoon depicting VSN dichotomy from stem cell/ neuronal progenitor stage and dynamic transcriptomic expression of stage specific transcription factors. D) The single cell pseudotime trajectory of Seurat object2 predicted by Monocle Seurat wrapper and visualized by UMAP. Cells are ordered in pseudotime by choosing Ascl1+ cells as root node and colored in a gradient from purple to yellow. D') Dynamic expression of small set of genes as a function of pseudotime.

\section{transcriptomes}

passed the quality control measures. Based on the expression of top 2000 highly variable features across the population, cells were clustered in Seurat object1 and visualized using 
uniform manifold approximation projection (UMAP) [26] (Fig. 1A). We identified the neuronal dichotomy (circled region in Fig. 1A) and annotated the cell clusters that belong to stem cells, neuronal progenitors, immediate neuronal precursors and immature VSNs based on the following gene expression pattern: Sox2/Ascl1 - Neuronal progenitors; Neurog1/Neurod1 - Immediate neuronal precursors; Gap43- immature VSNs; Meis2apical VSNs; Tfap2e- basal VSNs (Supplementary Fig. 1). We focused our further clustering and analysis only on Ascl1+, Neurod1+ and Gap43+ cells (circled in Fig. 1A) as our main interest is to characterize the mechanisms underlying the VSN cell fate specification. We subset these clusters into Seurat object2 (Fig. 1B) and re-clustered based on highly variable features among the selected cells. The expression pattern of known apical and basal specific genes as shown in the heatmap (Fig. 1B'), further corroborated the sc-RNA seq analyzed data with the literature $[11,14,20]$. Interestingly, Sox2 expression was found to be extended from the Ascl1+ progenitors' stage to Neurog1+ and to a certain extent of Neurod1+ neuronal precursors' stage (Fig. 1B', Fig. 2B), similar to what previously shown in the main olfactory epithelial studies [27, 28]. In addition, we found that Neurod1 expression, that starts along with Neurog1 after Ascl1, is retained beyond the dichotomy in both apical and basal VSN lineages (Fig. 1B', Fig. 2B). We labelled Neurog1+/Neurod1+ cells before the dichotomy as immediate neuronal precursors, whereas Neurod1+ cells in apical and basal lineages, after the dichotomy, as putative apical and basal neuronal precursors respectively.

We further implemented Monocle single cell trajectory analysis on Seurat object2 [29, 30]. In this analysis, we chose Ascl1 positive neuronal progenitors as a root node and rest of the single cells in Seurat object2 were ordered based on pseudotime, which is a unit of progress. This revealed a branched trajectory confirming the VSN dichotomy obtained from Seurat analysis (Fig. 1D, D').

\section{Differential gene expression identifies the expression of Notch signaling related genes at the VSN dichotomy.}




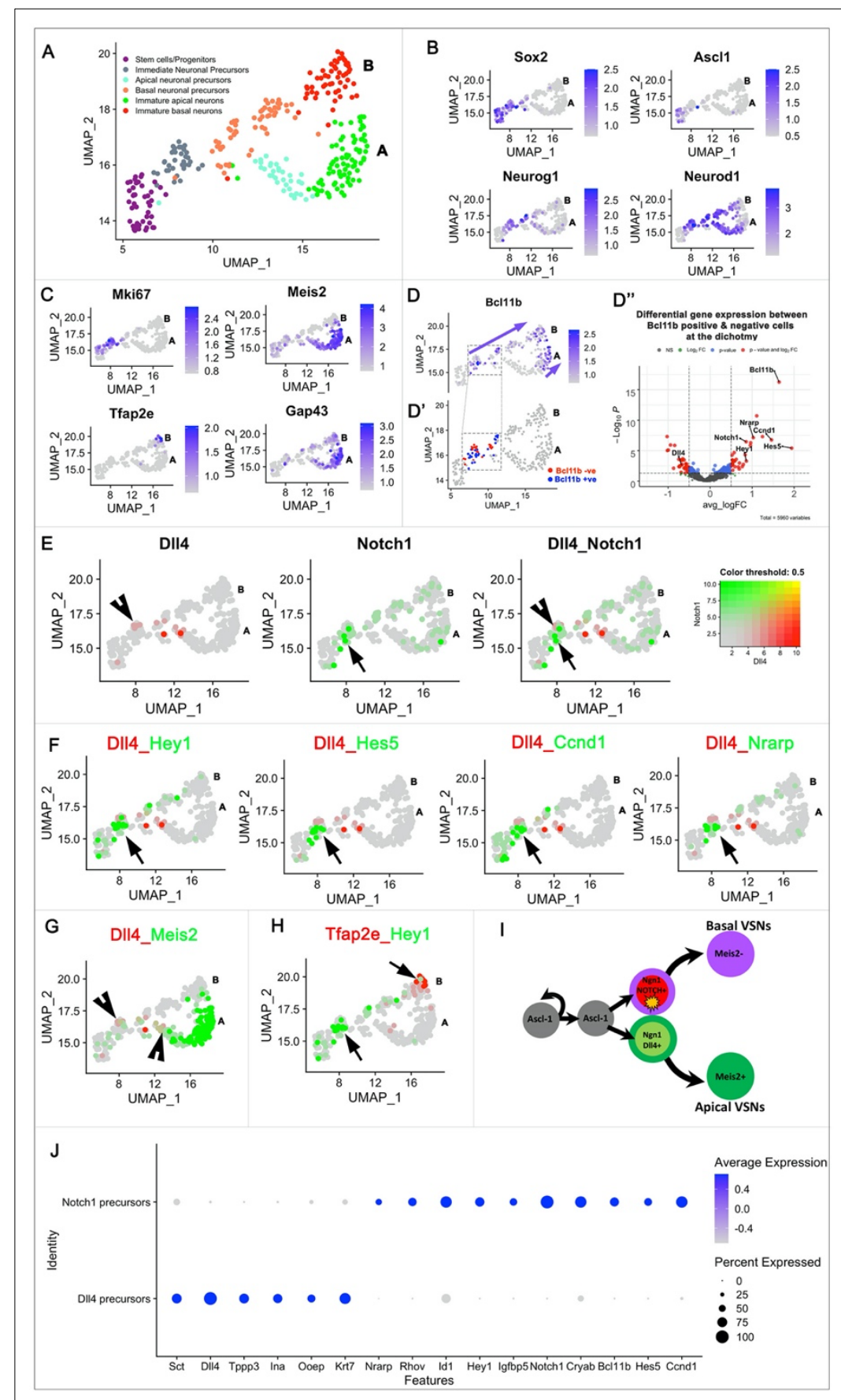

To further focus on the VSN dichotomy, we re-clustered Ascl1+ progenitors and VSNs' neuronal precursors to form Seurat object 3 (Fig. 2A). Feature plots of Ascl1, Sox2, Neurog1 and Neurod1 (Fig. 2B) identified the temporal transcriptional cascade as neuronal progenitors proliferate and differentiate into immediate neuronal precursors. Further analysis showed, as expected [11, 14], a distinct expression of the transcription factor Meis2 in post-mitotic (Ki67 negative) cells acquiring apical VSNs' identity and AP- $2 \varepsilon$ expression specific to maturing (GAP43 positive) basal VSNs (Fig. 2C). As previously known [14], the expression of basal specific gene AP- $2 \varepsilon$ appears

Figure 2: sc-RNA seq analysis identifies Notch1-DII4 signaling in establishing VSN dichotomy. For all the plots in this figure panel, alphabets A and B denotes apical and basal VSN branches. A) UMAP dimension plot of Seurat object3 that is specifically focusing on the VSN dichotomy. B, C) Feature plots of known marker genes that are specific to neuronal progenitors, precursors and immature apical and basal VSNs. D) Feature plot of Bcl11b expression at the VSN dichotomy. Arrow mark highlights continuous gene expression in the basal VSN branch, whereas it appears at a later stage in the apical VSN branch. D') Feature plot highlighting Bcl11b positive and negative cells at the dichotomy considered for differential gene expression analysis. D') Volcano plot highlighting Notch related genes differentially expressed between Bcl11b positive and negative clusters. E) Feature plots of Dll4 in red (arrowhead) and Notch1 in green (arrow) show their complimentary expression at the VSN dichotomy. F) Co-expression feature plots of DII4 vs downstream Notch signaling targets like Hes5, Hey1, Nrarp and Ccnd1 (follow arrow marks) show that active Notch signaling happens transiently at the dichotomy and early stages of basal VSN trajectory. G) Co-expression feature plot of DII4 vs Meis2 (arrowhead) shows DII4 expression towards apical VSN branch H) Co-expression feature plot of Hey1 vs Tfap2e (arrows) shows active Notch signaling hapenning towards basal VSN branch. I) Summary schematic showing the summary of Notch1-DII4 signaling required for establishing VSN dichotomy. J) Dot plot of a few selected genes that are differentially expressed between DII4 and Notch1 positive cluster at the VSN dichotomy. 
at later stages of maturation compared to apical specific Meis2 transcription factor. Following the indication of a previous study [11] that Bcl11b is a key gene in controlling cell fate choice of apical and basal VSNs, we looked at the spatial expression pattern of Bcl11b in Seurat object 3 (Fig. 2D). Overall, initial Bcl11b expression was seen after Ascl1 expression ended and after Neurog1/Neurod1 neuronal precursor stage started. On the basal VSN trajectory, Bcl11b expression was detected as a continuum from the dichotomy throughout the immature VSNs' stage, whereas on the apical VSNs' trajectory, Bcl11b mRNA expression was not detected until early stages of maturation (Arrows in Fig. 2D). Based on this observation, we manually clustered Bcl11b positive and negative cells specifically at the dichotomy (see boxed area in Fig. 2D and 2D'). Performing differential gene expression
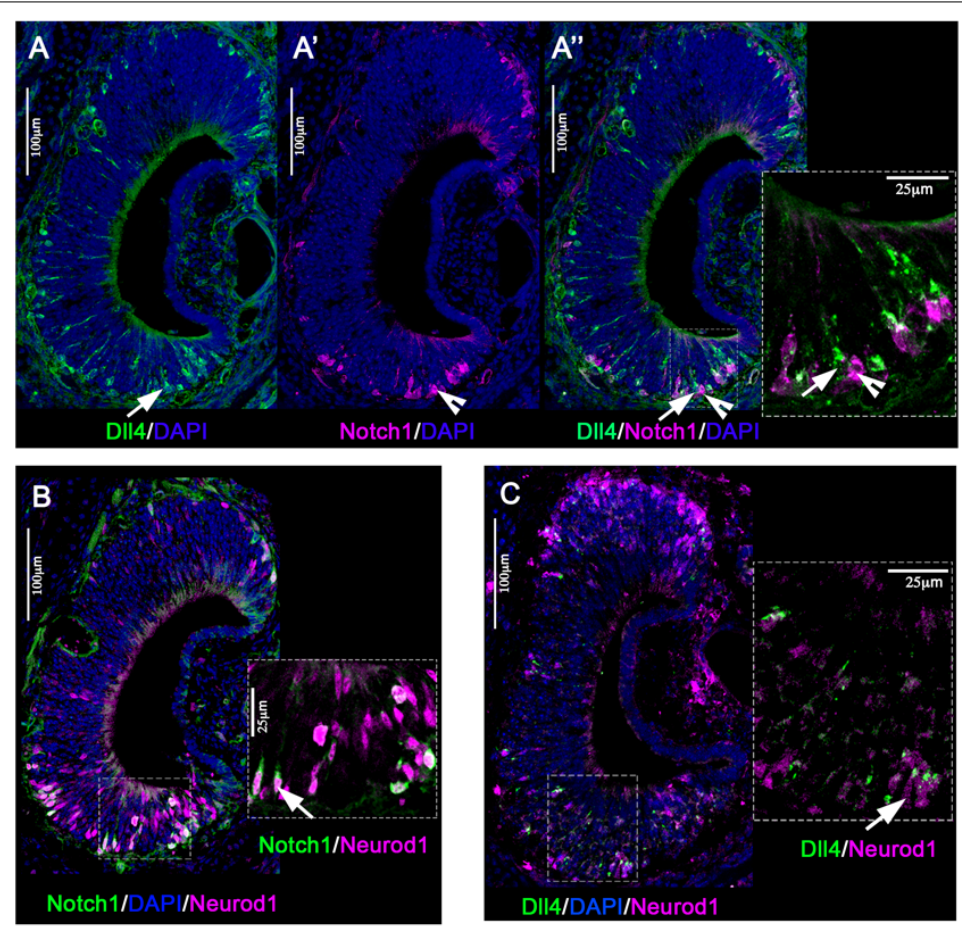

Figure 3: Notch1 and DIl4 immunoreactivity in Neurod+ cells. A) Double immunofluorescence of Dll4 in green and Notch1 in magenta shows expression of Notch ligand and receptor in marginal zones and basal regions of the VNO at postnatal day1. A" inset shows DIl4 (arrow) and Notch1+ (arrowhead) cells lying close to each other. B) Double immunofluorescence of Notch1 in green and Neurod1 in magenta shows (arrow) that Notch1 expression is mostly seen in Neurod1 stage. C) Double immunofluorescence of DIl4 in green and Neurod1 in magenta shows (arrow) that Dll4 ligand expression is mostly seen in Neurod1 stage.

analysis between Bcl11b positive and negative cells specifically at the dichotomy (Fig. 2D'), we found Notch1receptor as an enriched gene in the Bcl11b positive cluster, whereas DII4, which is a Notch ligand, is enriched in the Bcl11b negative cluster (Fig. 2D").

Moreover, we also identified Hes5, Hey1, Nrarp, and Ccnd1 enriched in the Bcl11b positive cluster (Fig. 2D"), which along with Bcl11b are known to be downstream Notch signaling targets [31-34]. Spatial colocalization plots of Notch1 and DII4 highlighted their mutually exclusive expression in cells before the neuronal dichotomy was established (Fig. 2E). Even though Notch mRNA could be detected in both apical and basal maturing 
VSNs after the dichotomy (Fig. 2E), Notch downstream targets like Hey1, Hes5, Nrarp and Ccnd1 appeared to be expressed only in Notch1+ but not DII4+ cells (Fig. 2F) before the VSN bifurcation and in the early stage basal VSN trajectory (Fig. 2F). In addition, DII4 ligand expression was found along with Meis2, which is an apical specific VSN marker, whereas downstream Notch signaling target Hey1 was found to be expressed along the basal VSN trajectory before Ap2 $\varepsilon$ (see colocalization feature plots in Fig. 2G, H). These gene expression plots suggest that Notch signaling is selectively activated in the cells acquiring basal identity during the establishment of the apical vs basal dichotomy but not after. Notably, many cells that are negative for Notch downstream target genes at the dichotomy are positive for Numb expression, suggestive of a post-translational Notch degradation after the dichotomy (Supplementary Fig. 2) [35, 36].

To further validate the biological relevance of differential Notch-Delta expression in the developing VSNs, we performed immunofluorescence staining against DII4, Notch1, and Neurod1 on vomeronasal tissue at postnatal day 1 (P1). Double immunofluorescence of DII4 and Notch1 confirmed the presence of immunodetectable Notch and Delta limited to Neurod1 positive precursors in the VNO (Fig. 3A, B, C). This analysis also highlighted DII4+ and Notch1+ cells lying close to each other (Fig. 3A"). These data suggest that differential Delta-Notch expression and activation of Notch signaling, in newly formed VSNs' precursors, could play a role in establishing a binary differentiation of VSNs.

\section{Conditional lineage tracing confirms the formation of Notch1+ and DII4+ cells from} Ascl1+ progenitors.

Temporally controlled genetic lineage tracing of stem cell/progenitors can be used to follow proliferation and differentiation dynamics [37]. We performed conditional lineage

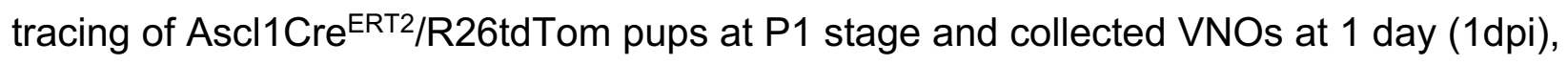
3 days ( $3 \mathrm{dpi}$ ) and 7 days post injection ( $7 \mathrm{dpi}$ ) (Fig. 4A). Further immunostainings were done to answer 2 main questions: 1) How long does it take for Ascl1 progenitors to become post mitotic? and 2) Do Ascl1+ progenitors give rise to both DII4+ and Notch1+ VSNs' precursors? 
After a single tamoxifen injection, we observed that the number of Ascl1Cre traced cells increased steadily from $30 \pm 4$ cells at 1 dpi to $142 \pm 25$ cells at 3 dpi and $225 \pm 58$ cells at $7 \mathrm{dpi}$ as expected ( $n=3$ at each stage and values in $\pm S D$ ). However, the percentage of proliferative traced cells (Ki67+/tdTom) decreased from $93 \pm 2.9 \%$ at 1 dpi to $12 \pm 1.7 \%$ at $7 \mathrm{dpi}(\mathrm{n}=3$ at each stage and values in \pm SD) (Fig. 4B). These data suggest that Ascl1+ transit amplifying cells [38] of the VNO go through multiple rounds of cell division before becoming postmitotic neurons.

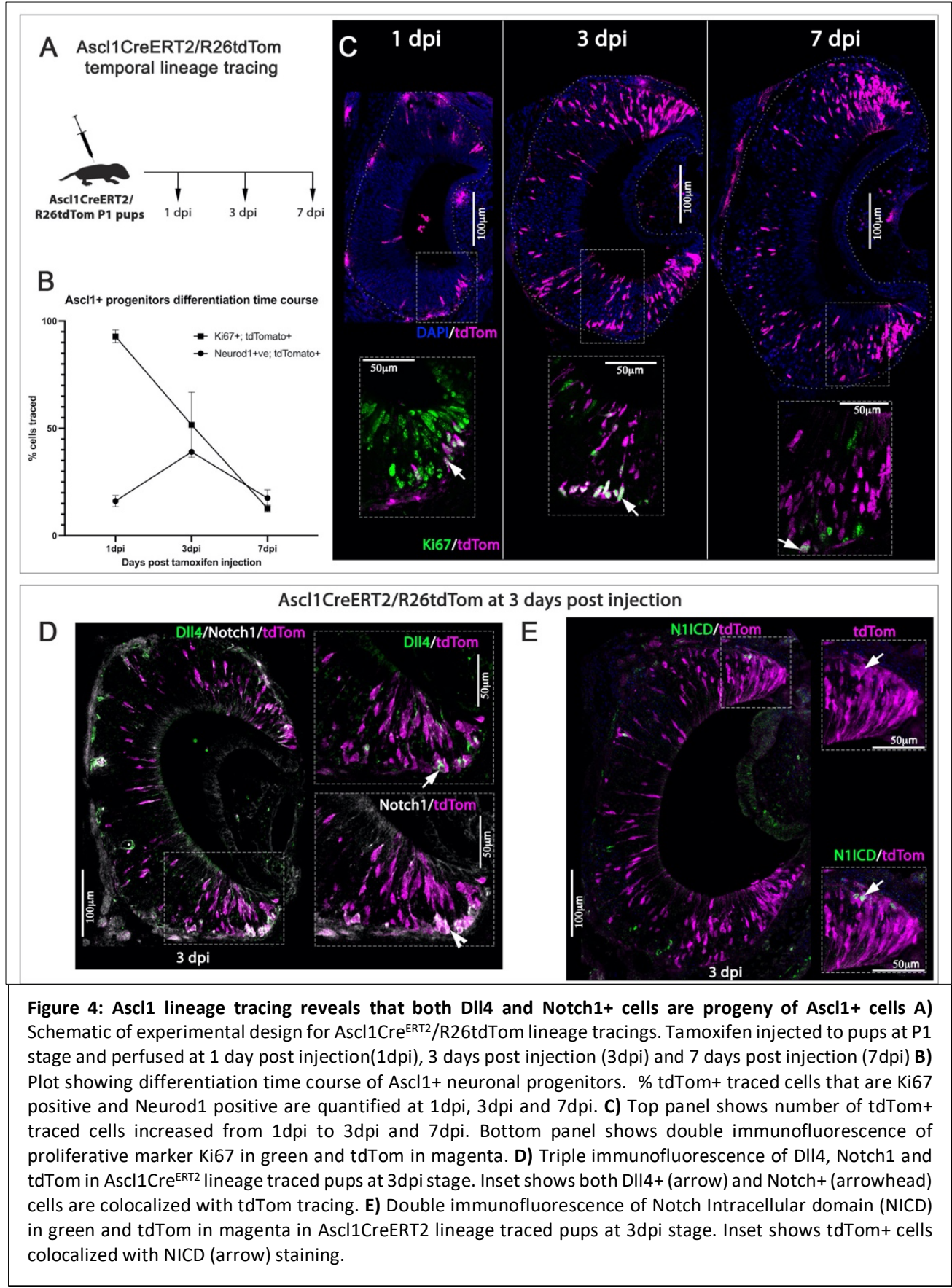


Analyzing the expression of Neurod1, which is a marker of cells undergoing differentiation (Fig. 1B', 2B), we observed that Ascl1 traced cells, positive for Neurod1 immunoreactivity, transiently increased from $16 \pm 2.6 \%$ at $1 \mathrm{dpi}$ to $39 \pm 0.6 \%$ at 3 dpi and then decreased to $17 \pm 3.9 \%$ at $7 \mathrm{dpi}$ as they start to mature $(n=3$ at each stage and values in \pm SD) (Fig. 4B). These results indicate that 1 week after temporally controlled Ascl1 lineage tracing, most of the cells become post mitotic and committed towards neuronal differentiation.

To further confirm the suitability of the experimental paradigm, we quantified the number of Ascl1Cre ${ }^{\mathrm{ERT} 2}$ traced cells that entered either the apical Meis2+ or basal Meis2(See Fig. 2C) differentiation programs. Meis2/tdTom quantifications at $7 \mathrm{dpi}$ indicated that $51 \%$ of the traced cells entered the apical VSN differentiation program while the rest of the Meis2- traced accessed the basal VSN lineage (Fig. 5B, D). Overall, this experiment indicates that 7 days after Ascl1Cre ${ }^{\mathrm{ERT} 2}$ recombination is sufficient time interval to analyze the phenotypes emerging after genetic manipulations of vomeronasal progenitors.

In addition to this, we also performed Dll4/Notch1/tdTom triple immunofluorescence staining for Ascl1Cre ERT2/R26tdTom tracing at 3dpi (Fig. 4D). As expected, we saw Dll4/tdTom and Notch1/tdTom double positive cells mostly in the marginal zones and basal zones of the vomeronasal sensory epithelium, where most of the postnatal neurogenesis takes place. Moreover, staining against cleaved Notch Intracellular Domain (NICD) confirmed Ascl1+ traced progeny undergoing active Notch signaling at $3 \mathrm{dpi}$ (Fig. 4E). In summary, these results along with the Neurod1 immunofluorescence staining (Fig. 3B, C; Fig 4B) suggest that Ascl1+ progenitors divide further to give rise to Neurod1+ precursors that transiently express either DII4 ligand or Notch1 receptor that can undergo active Notch signaling.

In the absence of active Notch signaling maturing VSNs default to the apical cell fate.

To test the role of Notch signaling in establishing the differentiation of VSNs, we decided to conditionally knock out Notch1 receptor from Ascl1+ progenitor stage onwards. We chose Ascl1Cre ${ }^{\text {ERT2 }}$ driver, as both Notch1 and DII4 expression start immediately after Ascl1 stage and around the Neurog1/Neurod1 stage (see Fig. 2B, 2E). We induced Cre 
recombination at $\mathrm{P} 1$ in both Ascl1Cre ERT2/R26tdTom $^{+/}$controls and Ascl1Cre ${ }^{\text {ERT2} / N o t c h 1 ~} 1$ fl/fl/R26tdTom ${ }^{+/}$pups and analyzed at 7 days post injection. In control pups, 1 week after lineage tracing, $51 \%$ of the traced cells were found positive for the apical marker Meis2+, while 49\% cells were Meis2- (Fig. 5B, D). Analyzing the

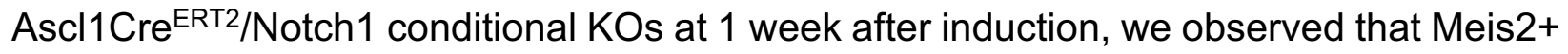
apical population significantly increased from $51 \%$ in control to $67 \%$ in the conditional $\mathrm{KO}$, whereas Meis2- population decreased proportionally. A deviation from the basal differentiation program was further confirmed with Ap2 $\varepsilon$ immunostaining which showed around $50 \%$ reduction in the maturing basal VSNs 1 week after conditional mutagenesis (Fig. 5D'). These results suggest that active Notch signaling, via Notch1 receptor, is essential to trigger the activation of the basal differentiation program.

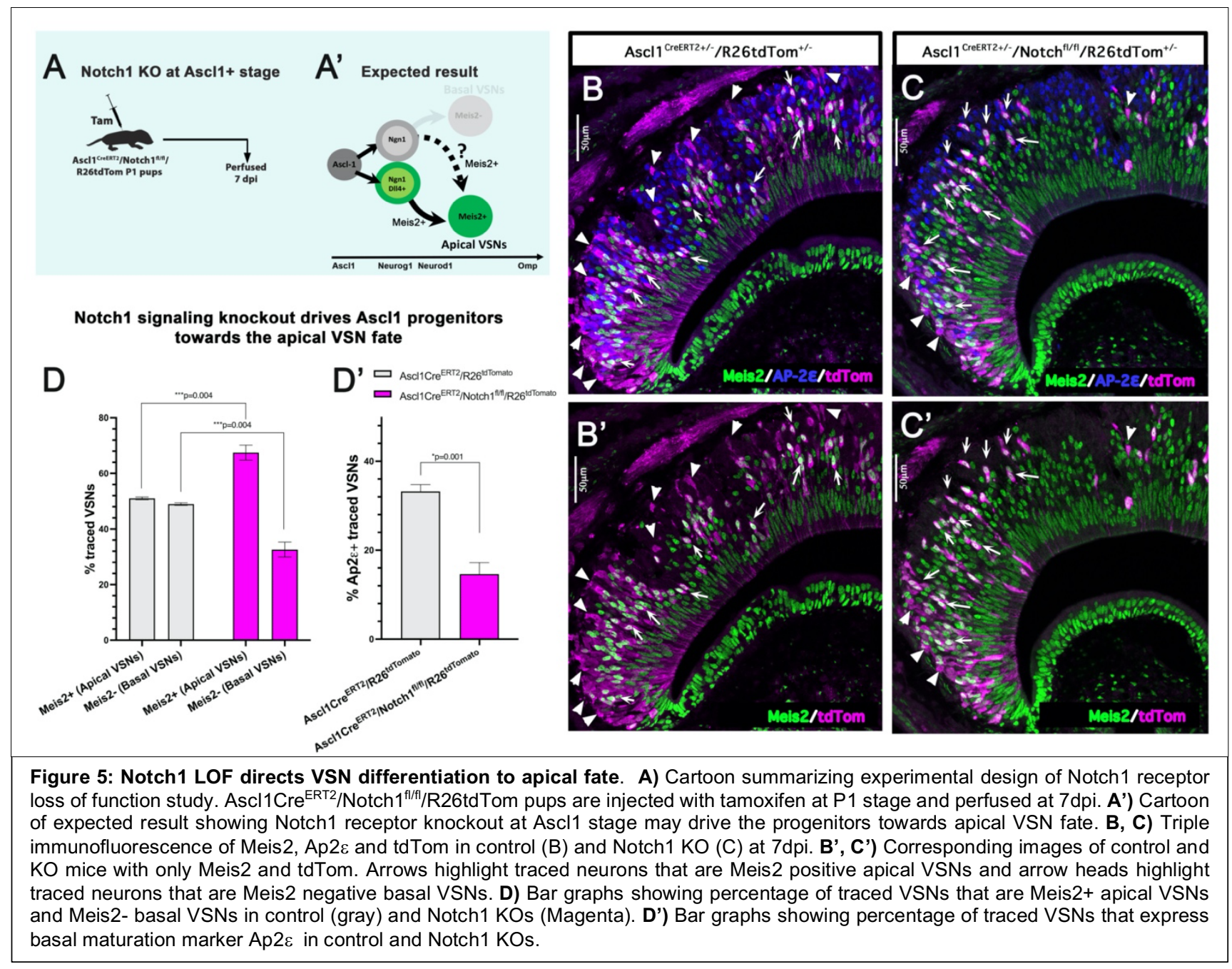




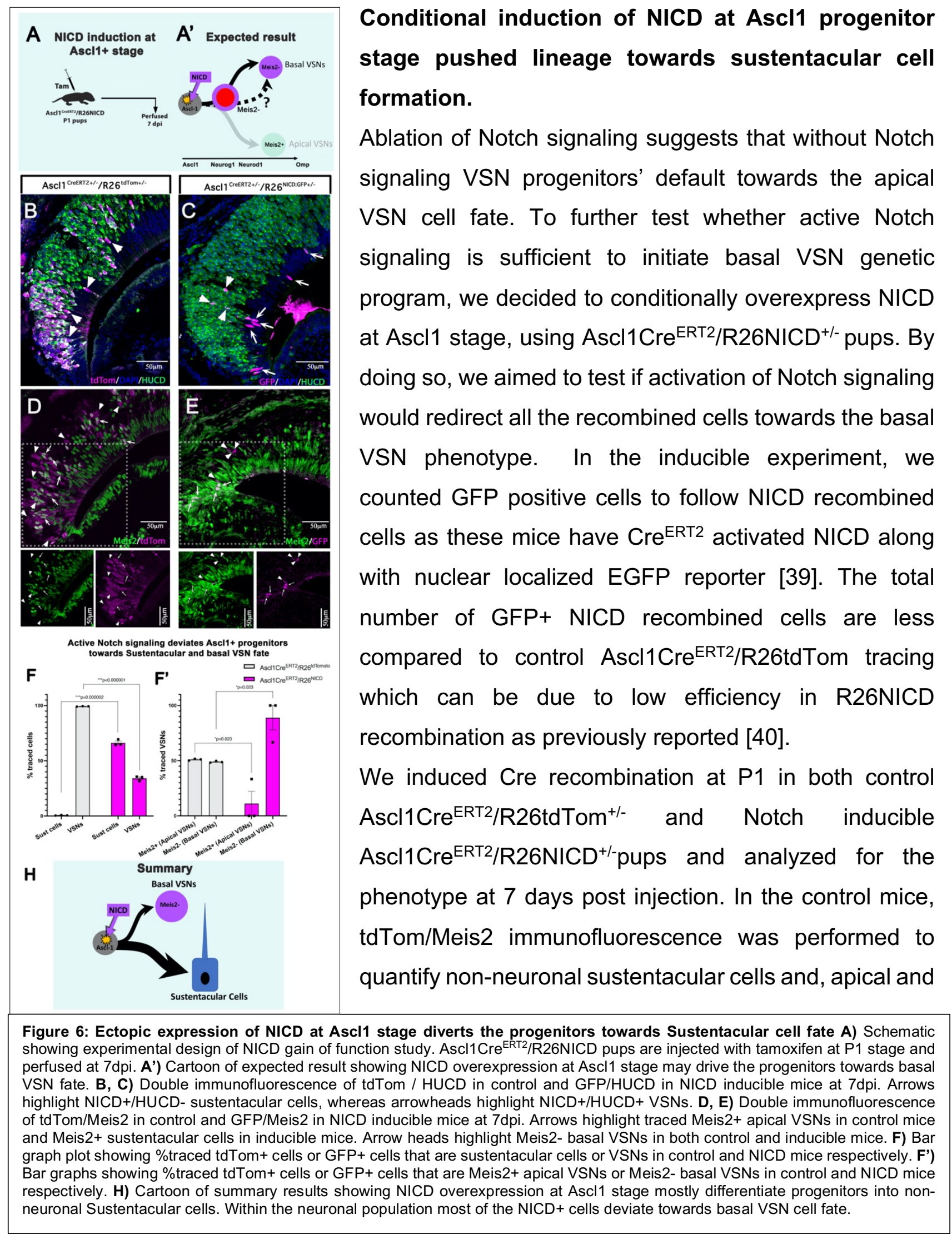


basal VSNs. While Meis2 is expressed in both apical VSNs and sustentacular cells, the morphology of the tdTom traced cell is used to distinguish between sustentacular cell and apical VSN. The analysis showed that at $7 \mathrm{dpi}$ in control tracing, 99.3\% of traced cells became VSNs out of which, 50 \% expressed the apical marker Meis2 and less than 1\% of the traced cells were non-neuronal Sustentacular cells (Fig. 6B, F).

However, in Ascl1Cre ERT2/R26NICD ${ }^{+/-}$pups, we found, to our surprise, conditional NICD overexpression in Ascl1+ progenitors mostly induced the formation of non-neuronal Sustentacular cells. We performed GFP/Meis2/HUCD triple immunofluorescence to distinguish among sustentacular cells and, apical and basal VSNs in the NICD inducible pups. As HUCD is only expressed in neuronal population [41], we considered Meis2+/HUCD- as non-neuronal sustentacular cells, Meis2+/HUCD+ as apical VSNs and Meis2-/HUCD+ as basal VSNs. The analysis in the inducible mice showed that $66.6 \%$ of the NICD: EGFP population became sustentacular and only $32.3 \%$ of the recombined cells were found to be VSNs (Fig. 6C, F). Within the recombined neuronal population, nearly all of them were negative for the apical marker Meis2 (Fig. 6F'). These data suggest that sustained activation of NICD in Ascl1 progenitor mostly deviates the neurogenic progenitors towards Sustentacular cells differentiation. However, the neurons that formed from NICD+ Ascl1 progenitors resulted to be Meis2 negative, therefore not committed to apical fate.

Conditional induction of NICD at Neurog1 precursors stage leads to reduction in Meis2+ apical VSN formation. 


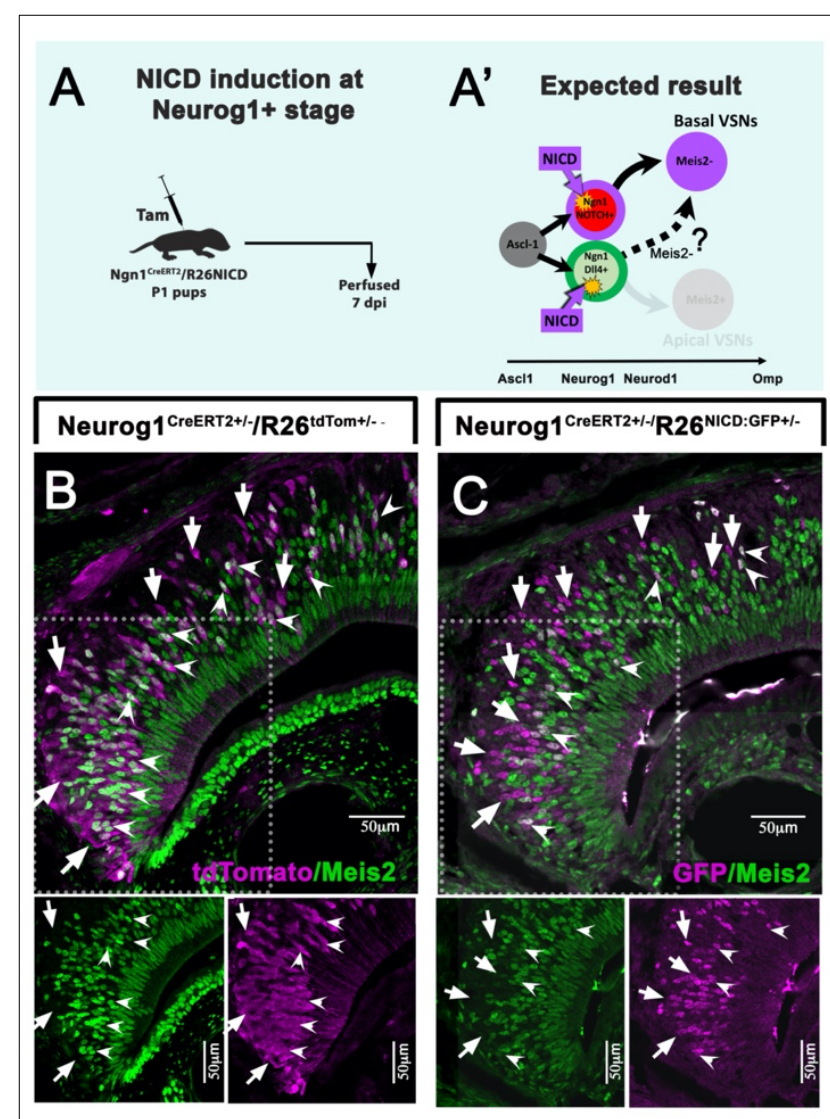

Activative Notch signaling in Neuorg1 precursors is sufficient to induce basal VSNs' program D
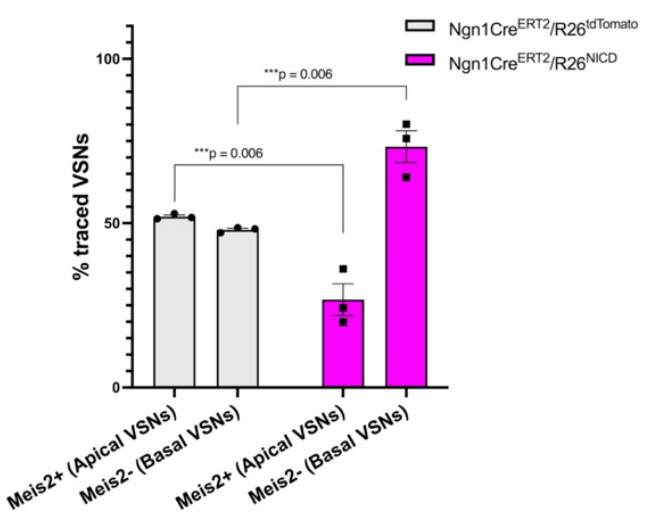

Figure 7: Ectopic expression of NICD at Neurog1 stage diverts the neuronal precursors towards basal VSN fate A) Schematic showing experimental design of NICD gain of function study. Neurog1Cre $e^{E R T 2} / R 26 N I C D$ pups are injected with tamoxifen at P1 stage and perfused at $7 \mathrm{dpi}$. A') Cartoon of expected result showing NICD overexpression at Neurog1 stage may drive the progenitors towards basal VSN fate. B, C) Double immunofluorescence of tdTom/Meis2 in control and GFP/Meis2 in NICD inducible mice at $7 \mathrm{dpi}$. Arrows highlight traced Meis2- basal VSNs and arrowheads highlight Meis2+ apical VSNs in both control and inducible mice. D) Bar graphs showing \%traced tdTom+ cells or GFP+ cells that are Meis2+ apical VSNs or Meis2- basal VSNs in control and NICD mice respectively.
Based on the intriguing results obtained at Ascl1 stage, we decided to test if later NICD activation, in committed (Neurog1+) neuronal precursors, could give us a clearer readout of the role of NICD in controlling the apical-basal dichotomy. Moreover, the Neurog1/Neurod1 stage is coincident to the stage when Notch signaling is more active and the dichotomy appears to be established (Fig 2 B, D, E, G).

We induced Cre recombination at $\mathrm{P} 1$ in both control Neurog1Cre ERT2/R26tdTom ${ }^{+/-}$and Notch inducible Neurog1Cre ERT2/R26NICD $^{+/-}$pups and analyzed 7 days post injection. We counted tdTomato+ cells and NICD: EGFP+ cells to follow the recombined cells in control and inducible R26NICD mice respectively. In control tracing experiment, Meis2/tdTom analysis at 7 dpi showed around $52 \%$ of the cells expressed apical VSN marker (Meis2+) while the rest of the $48 \%$ population acquired basal (Meis2-) VSN fate (Fig. 7 B, D). Notably, within the Meis2- cells that enter the basal program, 96.57\% expressed the transcription factor AP-2 $\varepsilon$ suggesting that they arrive to the basal maturing stage.

$$
\text { However, in the }
$$

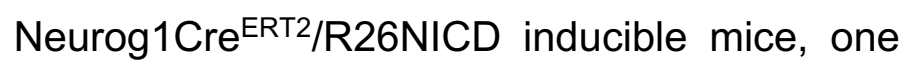
week after constitutive NICD overexpression, we found that Meis2- putative basal VSN population significantly increased to $\sim 73 \%$ (Fig. 7 C, D). Interestingly, within this Meis2- population, only $56.4 \%$ expressed the basal maturation marker 
AP-2ع. These data suggest that induction of Notch signaling at Neurog1 stage is sufficient to deviate the newly formed VSNs' away from the apical (Meis2+) differentiation program, however, prolonged NICD signaling might also interfere with normal basal VSN maturation.

\section{Discussion:}

Interaction of extrinsic and intrinsic regulators is important for neural stem cells/ progenitors to make cell fate decisions and give rise to specific neuronal cell types. The VNE of mice is composed of V1R+/Gai2+ apical and V2R+/Gao+ basal VSN sub types. Correct development and function of these two main types of vomeronasal neurons is important for social, sexual, maternal and predator avoidance behaviors of rodents [1619]. However, Bcl11b is the only known intrinsic regulator till now shown to affect the cell fate decision in the VNO [11]. In the current study, for the first time we identified the role of DII4-Notch1 signaling in the cell fate specification of apical and basal VSNs. In addition, research from $\mathrm{T}$ cell development previously reported $\mathrm{Bcl} 11 \mathrm{~b}$ as direct downstream Notch signaling target gene [42], thus relating our current study to the Bcl11b role in the VNO.

In this study, sc-RNA seq analysis of adult VNO at the neuronal dichotomy identified DII4 ligand and Notch1 receptor in the apical and basal committed neuronal precursors respectively. In addition, immunofluorescence data along with the conditional Ascl1Cre ${ }^{\text {ERT2 }}$ lineage tracing also confirmed that both Notch1+ and Dll4+ cells are the progeny of Ascl1 cells and are expressed at Neurod1 stage, suggesting a potential role for DII4-Notch1 signaling in establishing the VSN dichotomy. Previous studies also highlighted the expression of Notch1 and DII4 in developing VNO at different ages [43, 44].

Notch is an evolutionarily conserved juxtracrine signaling pathway associated with inhibitory interactions/lateral inhibitions that can determine cell fates among two juxtaposed differentiating cells $[45,46]$. Notch inhibitory ligand-receptor interactions rely on non-symmetric expression of a Notch transmembrane receptor and a Notch ligand between neighboring cells. Notch activation in a cell prevents it from assuming the same cell fate as the neighboring cell expressing the Notch ligand. Notch paralogs (Notch1, Notch2, Notch3, Notch4), three Delta-like ligands (DII1, DII3, DII4) and two Jagged-like 
ligands (Jagged1, Jagged2) have been found in mammals [47]. In this study, we performed LOF and GOF experiments at Ascl1+ neuronal progenitor stage and Neurog1+ neuronal precursor stage to probe the necessity and sufficiency of Notch signaling in establishing neuronal diversity in the VNO. In the LOF strategy, Notch1 receptor knockout from Ascl1+ neuronal progenitor stage onwards caused most of the neurons to enter the apical (Meis2+/V1R/Gai2) differentiation program. This suggests that neuronal progenitors are by default fated towards apical VSN type if not instructed otherwise by Notch mediated transcriptional regulation. Vomeronasal precursors with active Notch signaling express $\mathrm{Bcl11b}$ [11] and then AP-2 $\varepsilon$ at a later maturation stage [14]. From our single cell seq analysis, we found that Ascl1+ progenitors don't express Notch1 receptor. In line with this, Notch1 ablation at Ascl1 stage does not affect the neurogenic process, but the cell fate choice of VSNs.

Notch pathway has been extensively shown to be important for promoting gliogenesis and for maintaining glial function in rodents [48-51]. Moreover, ectopic activation of Notch signaling has also been previously shown to be able to alter cell fate [52]. Ectopic expression experiments of transcription factors at different maturation stages of neurons have revealed that the dynamic changes in the chromatin landscape play a key role in restricting the phenotypic plasticity of the cells [53]. In line with this, our conditional ectopic NICD GOF experiments in the VNO gave distinct phenotypes based on the timing of Cre recombination. In fact, NICD activation in Ascl1+ neural committed progenitors, which in control conditions mostly give rise to VSNs (see Fig. 6F), appears to be sufficient to induce their differentiation to Sustentacular cells. This non-neuronal phenotype may likely result from NICD-mediated negative regulation of pro-neurogenic transcription factors $[54,55]$ and by the activation of the alternative Sustentacular differentiation program. Notably, we already know that NICD expression in multipotent olfactory horizontal basal cells (HBCs) during regeneration is sufficient to induce Sustentacular cell differentiation and that Notch signaling is required for homeostasis of the Sustentacular cells $[48,56,57]$. Moreover, sc-RNA seq data also identified the expression of downstream Notch targets in the VNO Sustentacular population suggesting a role of Notch signaling in their formation (Supplementary Fig. 3). Notably, Ascl1+ progenitors have more restricted potency compared to the HBCs [58]. However, the 
phenotypic change of vomeronasal Ascl1+ progenitors in response to NICD implies that, at this stage, the chromatin landscape is still plastic enough to be rearranged by active Notch signaling[53, 59].

On the other hand, NICD activation at Neurog1 stage, did not convert neuronal cells to Sustentacular cells, but increased the proportion of Meis2- basal VSNs. This suggests that at Neurog1 stage, the VSNs' precursors reach a level of commitment at which the chromatin landscape is no longer permissive [53] for Notch-mediated reprograming from neurons to glia/Sustentacular cells. However, at this stage Notch signaling is sufficient to inhibit the progression of the Meis2+ apical VSN program and to divert the neuronal precursors towards the basal VSN fate. One interesting aspect with the ectopic GOF experiments is the relation of NICD with Meis2 expression. Meis2 is expressed in both apical VSN lineage and non-neuronal Sustentacular cells. Ectopic NICD expression at Ascl1 stage not only converted them into Sustentacular cells, but also maintained their Meis2 expression, whereas NICD overexpression at Neurog1 stage repressed apical VSN specific Meis2 expression diverting them into basal lineage. This suggests that NICD complex follows different downstream neuronal and sustentacular differentiation pathways depending on the developmental stage of the cell.

From our sc-RNAseq data (see Fig. 2E, 2G) and Ascl1Cre ERT2/R26tdTom control tracing (see Fig. 4E) we know that, in physiological conditions, active Notch signaling is temporally restricted to Notch1+ cells when the apical-basal dichotomy is established. After Neurog1Cre driven NICD expression, we found that even though more VSNs deviate from the apical program, a smaller percentage of Meis2- cells (basal committed)

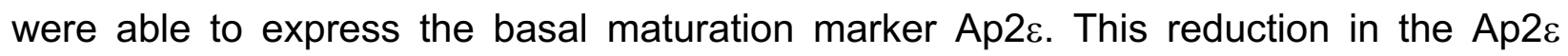
expression may likely result from the non-physiological timing/levels of NICD activation in the Neurog1 ${ }^{\text {CreERT2/R26NICD mice. }}$

Notch signaling role in cell fate specification is conserved in many neuronal and non-neuronal systems [60-63]. Further, research into Notch1-DIl4 signaling literature in retina and spinal cord pointed out Foxn4, a forkhead transcription factor to have a role in inducing Notch1- DII4 mediated signaling [63-65]. In retina, Foxn4 is important in diverting the retinal progenitor cell fate towards amacrine cells and horizontal cells, whereas in spinal cord, Foxn4 is expressed in p2 progenitors that specifically give rise to V2a and 
V2b interneurons. In both systems, it has been shown that Foxn4 controls the expression of DIl4 and that Foxn4 mutants have aberrant cell fate phenotypes [63, 64]. Our sc-RNA seq data revealed Foxn4 mRNA expression partially overlapping to that of DIl4. We further corroborated co-expression via DII4/Foxn4 double immunofluorescence staining at E14 stage (Supplementary Fig. 4). The identification of DII4 and Foxn4 double positive cells suggested to us to test whether Foxn4 plays a role in apical vs basal VSN differentiation via DII4 ligand expression. However, Foxn4 null mutants still showed, as the wild type, detectable Dll4 expression at E14 stage, moreover, no obvious changes in the apical-basal VSN ratio was detected (Supplementary Fig. 4). These data suggest, that in the VNO, Foxn4 plays a dispensable role in inducing Dll4 expression.

Among Notch modulators, mammalian Numb and Numb-like proteins have been shown to repress Notch signaling by controlling NICD endosomal trafficking and degradation [66]. By taking a closer look at downstream Notch signaling targets like Hey1, Hes5 and, CyclinD1 vs Numb in sc-RNA seq data, we confirm that Numb expression negatively correlates with Notch-mediated signaling in the VNO (Supplementary Fig. 2). Notably these molecules play important roles in modulating the efficiency of Notch signaling but not Notch or delta expression.

By analyzing our single cell data, we observed that Notch and Delta mRNA appeared to be non-symmetrically distributed and mutually exclusive among cells even before the apical-basal VSNs' dichotomy is established (Fig. 2E). These data suggest that non-symmetric inheritance of RNA binding proteins or other post-transcriptional modulators [67-69] define the predetermination of the Notch and Delta neuronal precursors. In support of this idea, we observed that $\mathrm{KH}$ domain containing RNA binding protein (RBP) Oocyte expressed protein (Ooep), was significantly enriched in the $\mathrm{DIl4+/Bcl11b-cluster} \mathrm{but} \mathrm{not} \mathrm{Notch1/Bcl11b+} \mathrm{cluster} \mathrm{at} \mathrm{the} \mathrm{VSN} \mathrm{dichotomy} \mathrm{(Fig.} \mathrm{2).} \mathrm{Ooep,}$ also named as Floped/Moep19 is a maternal effect gene that is expressed in oocyte and regulates embryonic cell division via F actin cytoskeleton [70-73]. Moreover, in our data, even after the dichotomy, Ooep expression was found to persist only in the apical VSN branch along with Meis 2 which is one of the early apical VSN marker (Supplementary Fig. 5). As RBPs can also control cell fate choice by post-transcriptionally modulating mRNA and miRNA dynamics [74-76], it would be interesting to further study whether 
Ooep can work as intrinsic regulator of the Notch and Delta asymmetry in neuronal precursors.

Evolution of new sensory neuronal types in animals can have an important role in determining their social and environmental fitness by expanding their ability to detect, compute, and respond to new stimuli. Both V1R and V2R are functionally, and evolutionarily unrelated super families of receptors and previous studies highlighted the diversity of $\mathrm{V} 1 \mathrm{R}$ and $\mathrm{V} 2 \mathrm{R}$ positive $\mathrm{VSN}$ population across many vertebrate species [77, 78]. In most of the mammals e.g., horse, goat, musk shrew, common marmoset, dog, and cow, few to none $\mathrm{V} 2 \mathrm{R}+$ genes appear to be expressed. Notably rodents and opossum have strikingly expanded repertoire of functional V2R genes, moreover in these animals, specialized VSNs segregate into V1R+/Gai2+ apical and V2R+/Gao+ basal VSNs [10, 79]. As the current study has revealed the role of Notch signaling in the VSN cell fate specification, it would be interesting to further study the evolutionary development of mechanisms controlling Notch1-DII4 expression across different vertebrate species and see how these mechanisms influence the neuronal composition of the chemosensory epithelia across species. 


\section{Materials and Methods:}

Mouse lines: We purchased Ascl1Cre ${ }^{\mathrm{ERT} 2}$ (Asc/1tm1.1(Cre/ERT2)Jejo/J Stock No: 012882),

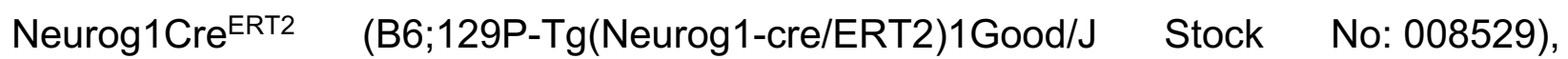
Notch1 ${ }^{\mathrm{fl} / \mathrm{fl}}\left(\mathrm{B} 6.129 \times 1-N o t c h 1^{\text {tm2Rko} / G r i d J} \quad\right.$ Stock No: 007181),

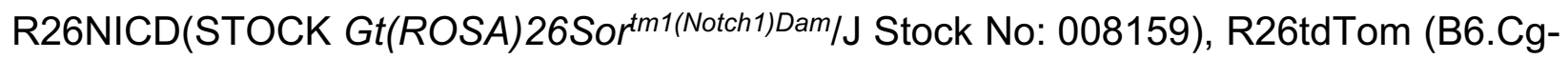
Gt(ROSA)26Sortm9(CAG-tdTomato)Hze/J, JAX stock \#007909) mouse lines from Jackson Lab. The Foxn4 $4^{+/ l a c z}$ mouse line was generated previously [80]. Genotyping was conducted following the suggested primers and protocols from JAX. Mice of either sex were used for immunohistochemistry and immunofluorescence experiments. All experiments involving mice were approved by the University at Albany Institutional Animal Care and Use Committee (IACUC).

\section{Single-Cell RNA Sequencing}

The vomeronasal organs of P60 C57BL/6J male mice were isolated and dissociated into single-cell suspension using neural isolation enzyme/papain (NIE/Papain in Neurobasal Medium with $0.5 \mathrm{mg} / \mathrm{mL}$ Collagenase A, $1.5 \mathrm{mM}$ L-cysteine and 100U/mL DNAse I) incubated at $37^{\circ} \mathrm{C}$. The dissociated cells were then washed with HBSS and reconstituted in cell freezing medium (90\% FBS, 10\% DMSO). Cells were frozen from room temperature to $-80^{\circ} \mathrm{C}$ at $\mathrm{a}-1{ }^{\circ} \mathrm{C} / \mathrm{min}$ freeze rate. The single cell suspension was sent to SingulOmics for high-throughput single-cell gene expression profiling using the 10x Genomics Chromium Platform. Data was analyzed using Seurat 3.15.

Quality control and cell clustering. Quality control (QC), and clustering and downstream analysis was performed using Seurat [3.15] package in R. Basic filtering was carried out where all genes expressed in $\geq 3$ cells and all cells with at least 200 detected genes were included. QC was based on number of genes and percent mitochondrial genes, where all cells that expressed $>9000$ genes and $>5 \%$ mitochondrial genes were not included in the analysis. After filtering, 10,582 cells were included for the clustering and analysis. Top 2000 highly variable genes across the population were selected to perform PCA and the first 35 principal components were used for cells clustering, which 
was then visualized using uniform manifold approximation projection (UMAP). Stem cells, neuronal progenitors, precursors and immature neuronal cell types were identified based on the expression of known genes. These cell types were specifically chosen to subset and top 2000 highly variable genes and top 15 principal components were used to cluster and create new Seurat object2. Similarly, Seurat object3 was created by focusing on clusters only at VSN dichotomy and top 20 principal components were used to cluster and visualize. All downstream analysis that identified Notch1-DII4 signaling were done on Seurat object3.

Pseudotime analysis of cell population spatial organization. To further confirm the VSN dichotomy, we used Monocle3 to perform pseudotime analysis, where Ascl1 positive cells were chosen as root node. We used Seurat wrappers package to directly convert Seurat object2 into cell data set format.

Tamoxifen Treatment: Tamoxifen (Sigma-Aldrich), CAS \# 10540-29-1, was dissolved in Corn Oil at $20 \mathrm{mg} / \mathrm{ml}$ concentration. For all tamoxifen inducible experiments, we injected tamoxifen once intraperitoneally at postnatal day 1 at a dose of $80 \mathrm{mg} / \mathrm{Kg}$ body weight and perfused at indicated postnatal days.

\section{Tissue Preparation}

Tissue collected were perfused with PBS followed by $3.7 \%$ formaldehyde in PBS. Noses were immersion fixed in $3.7 \%$ formaldehyde in PBS at $4^{\circ} \mathrm{C}$ for $1-2 \mathrm{hr}$ depending on the age. All samples were cryoprotected in $30 \%$ sucrose in PBS overnight at $4^{\circ} \mathrm{C}$ then embedded in Tissue-Tek O.C.T. Compound (Sakura Finetek USA, Inc., Torrance CA) using dry ice, and stored at $-80^{\circ} \mathrm{C}$. Tissue was cryosectioned using a CM3050S Leica cryostat at $16 \mu \mathrm{m}$ for VNOs and collected on VWR Superfrost Plus Micro Slides (Radnor, PA) for immunostaining. All slides were stored at $-80^{\circ} \mathrm{C}$ until ready for staining.

\section{Immunofluorescence}

Citrate buffer ( $\mathrm{pH}$ 6.0) antigen retrieval was performed [14], for all the antibodies indicated with asterisks $\left(^{*}\right)$. Primary antibodies and concentrations used in this study were, *Goat anti-AP-2ع (2ug/mL, sc-131393 X, Santa Cruz, Dallas, TX), Chicken antiGFP (1:3000, ab13970, Abcam), Rabbit anti-GFP (1:1000, A-6455, Molecular Probes, 
Eugene, OR), *Rabbit anti-Ki67 (1:1000, D3B5, Cell signaling Tech), *Mouse anti-Ki67 (1:500, 9449, Cell signaling Tech), "Mouse anti-Meis2 (1:500, sc-515470, Santa Cruz), Rabbit anti-Meis2 ( ${ }^{*}$ with antigen retrieval 1:1000 and without antigen retrieval 1:500, ab73164, Abcam), *Mouse anti-NeuroD1 (1:100, sc-46684, Santa Cruz), *Goat antiNeuroD1 (1:500, AF2746, R\&D Systems), *Rabbit anti-Notch1 (1:50, D1E11, Cell Signaling Tech), Goat anti-DII4 ('with antigen retrieval 1:50 and without antigen retrieval 1:25, AF1389, R\&D Systems), *Rabbit anti-NICD activated (1:75, D3B8, Cell Signaling Tech), "Mouse anti-DsRd (1:500, TA180084, Origene), *Rabbit anti-DsRd (1:500, 600-

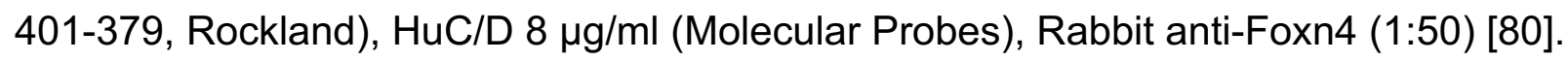

For chromogen-based reactions, tissue was stained as previously described [14]. Staining was visualized with the Vectastain $A B C$ Kit (Vector, Burlingame, $C A$ ) using diaminobenzidine (DAB); sections were counterstained with methyl green.

Species-appropriate secondary antibodies conjugated with either Alexa Fluor 488, Alexa Fluor 594, Alexa Fluor 568, Alexa Fluor 680 plus were used for immunofluorescence detection (Molecular Probes and Jackson ImmunoResearch Laboratories, Inc., Westgrove, PA). Sections were counterstained with 4',6'-diamidino-2phenylindole (DAPI) (1:3000; Sigma-Aldrich) and coverslips were mounted with FluoroGel (Electron Microscopy Services, Hatfield, PA). Confocal microscopy pictures were taken on a Zeiss LSM 710 microscope. Epifluorescence pictures were taken on a Leica DM4000 B LED fluorescence microscope equipped with a Leica DFC310 FX camera. Images were further analyzed using FIJI/ImageJ software.

\section{Experimental design, quantification, and statistical analyses of microscopy data.}

All data were collected from mice kept under similar housing conditions, in transparent cages on a normal $12 \mathrm{hr}$. light/dark cycle. Tissue collected from either males or females in the same genotype/treatment group were analyzed together unless otherwise stated; ages analyzed are indicated in text and figures. Measurements of VNE and cell counts were performed on confocal images of coronal serial sections immunostained for the indicated targets. Measurements and cell counts were done using ImageJ. The data are presented as mean \pm SEM unless otherwise specified. Prism 9.0.1 was used for statistical analyses, including calculation of mean values, and SEM. Two-tailed, unpaired t-test were 
used for all statistical analyses, and calculated p-values <0.05 were considered statistically significant. Sample sizes and p-values are indicated as single points in each graph and/or in figure legends.

\section{Author contributions:}

PEF conceptualized and designed the experiments. RRK designed, performed the experiments, and analyzed the data. MX designed experiments. SL performed the experiments and analyzed the data. EZT, SMH analyzed the data. JML performed the experiments. RRK and PEF wrote the manuscript and designed illustrations.

Funding: This publication was supported by the Eunice Kennedy Shriver National Institute of Child Health and Human Development of the National Institutes of Health under the Awards R15-HD096411 (P.E.F) and R01-HD097331/HD/NICHD (P.E.F), and by the National Institute of Deafness and Other Communication Disorders of the National Institutes of Health under the Award R01-DC017149 (P.E.F).

Mengqing Xiang's lab contribution was supported by the National Natural Science Foundation of China (81970794), Natural Science Foundation of Guangdong Province of China (2020A1515010328) and the Fundamental Research Funds of the State Key Laboratory of Ophthalmology, Sun Yat-sen University. 


\section{Supplementary figures:}

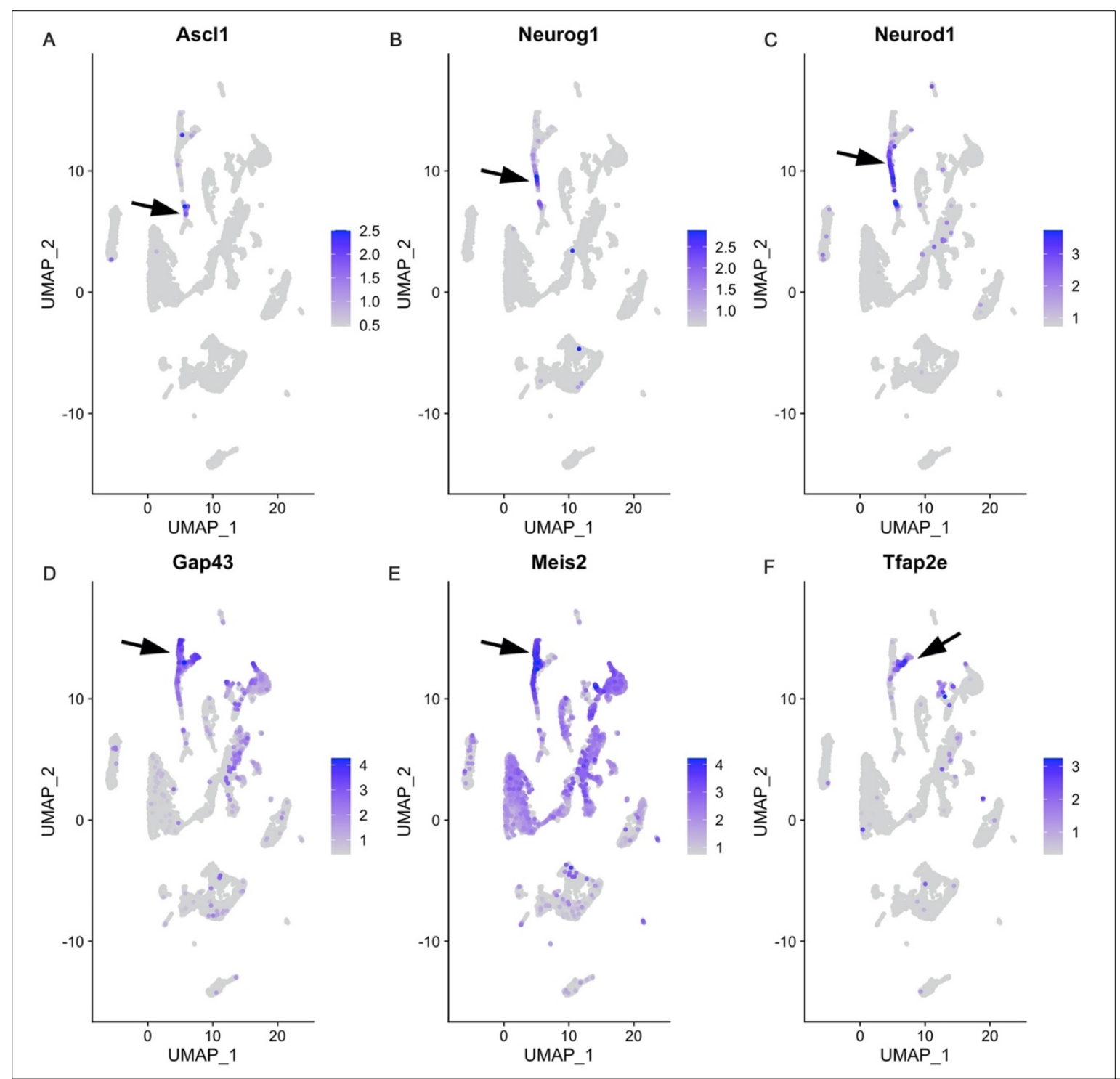

Supplementary figure 1: sc-RNA seq analysis identifies apical and basal VSN dichotomy. A-F) Feature plots of Ascl1, Neurog1, Neurod1, Gap43, Meis2 and Ap2 $\varepsilon$ genes that are specific to neuronal progenitors, precursors and immature apical and basal VSNs identify VSN dichotomy. Specific expression of the gens is highlighted by arrow. Both Meis2 and Tfap2e expression highlights apical and basal VSN branches respectively. 


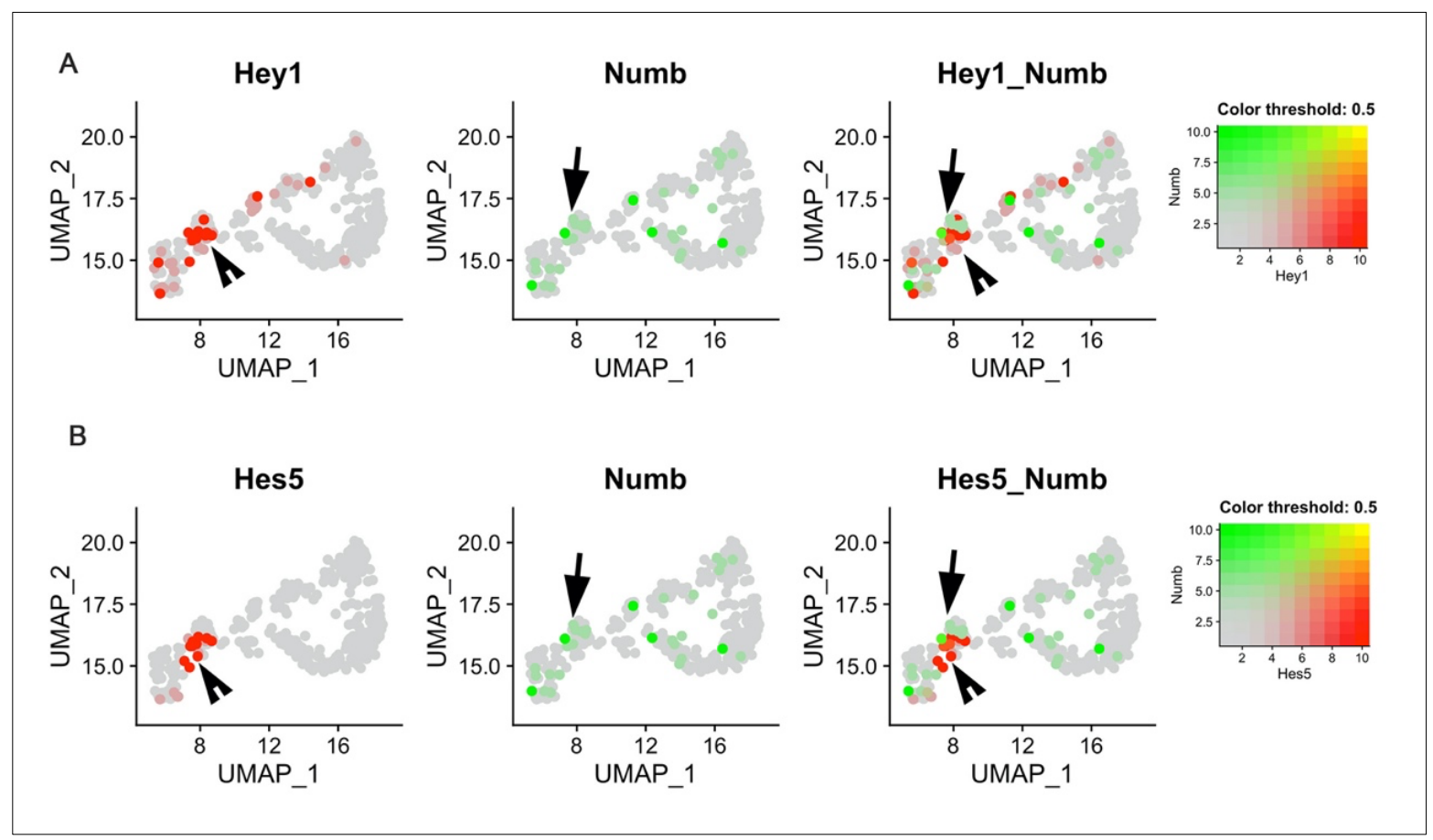

Supplementary figure 2: Expression of Numb and downstream Notch targets are mutually exclusive at the VSN dichotomy A, B) Blended feature plots of Notch target genes Hey1 and, Hes5 Vs Numb shows that Notch target genes are not expressed in similar populations along with Numb at the VSN dichotomy. 
bioRxiv preprint doi: https://doi.org/10.1101/2021.10.26.466003; this version posted October 26, 2021. The copyright holder for this preprint (which was not certified by peer review) is the author/funder. All rights reserved. No reuse allowed without permission.

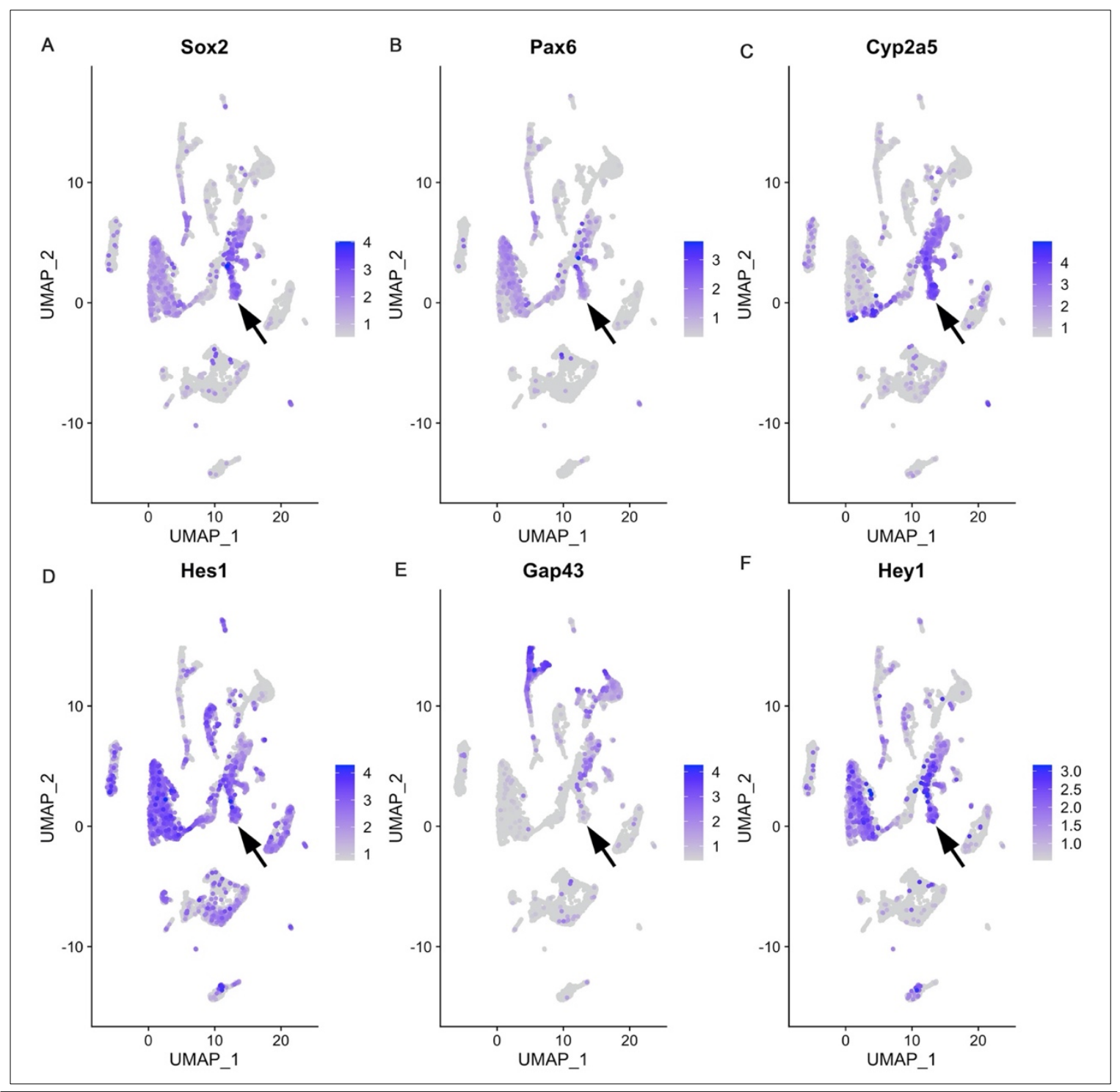

Supplementary figure 3: sc-RNA seq analysis reveals the expression of downstream Notch targets in Sustentacular cells. A-D) Feature plots of Sox2, Pax6, Cyp2a5 and, Hes1 shows their spatial expression patterns across all VNO cell types. Arrow highlights their specific expression in Sustentacular cells. E) Feature plot of immature neuronal marker Gap43 shows its lack of expression in the Sustentacular population (arrow mark). F) Feature plot of downstream Notch target Hey1 shows its expression in the Sustentacular population. 
bioRxiv preprint doi: https://doi.org/10.1101/2021.10.26.466003; this version posted October 26, 2021. The copyright holder for this preprint (which was not certified by peer review) is the author/funder. All rights reserved. No reuse allowed without permission.

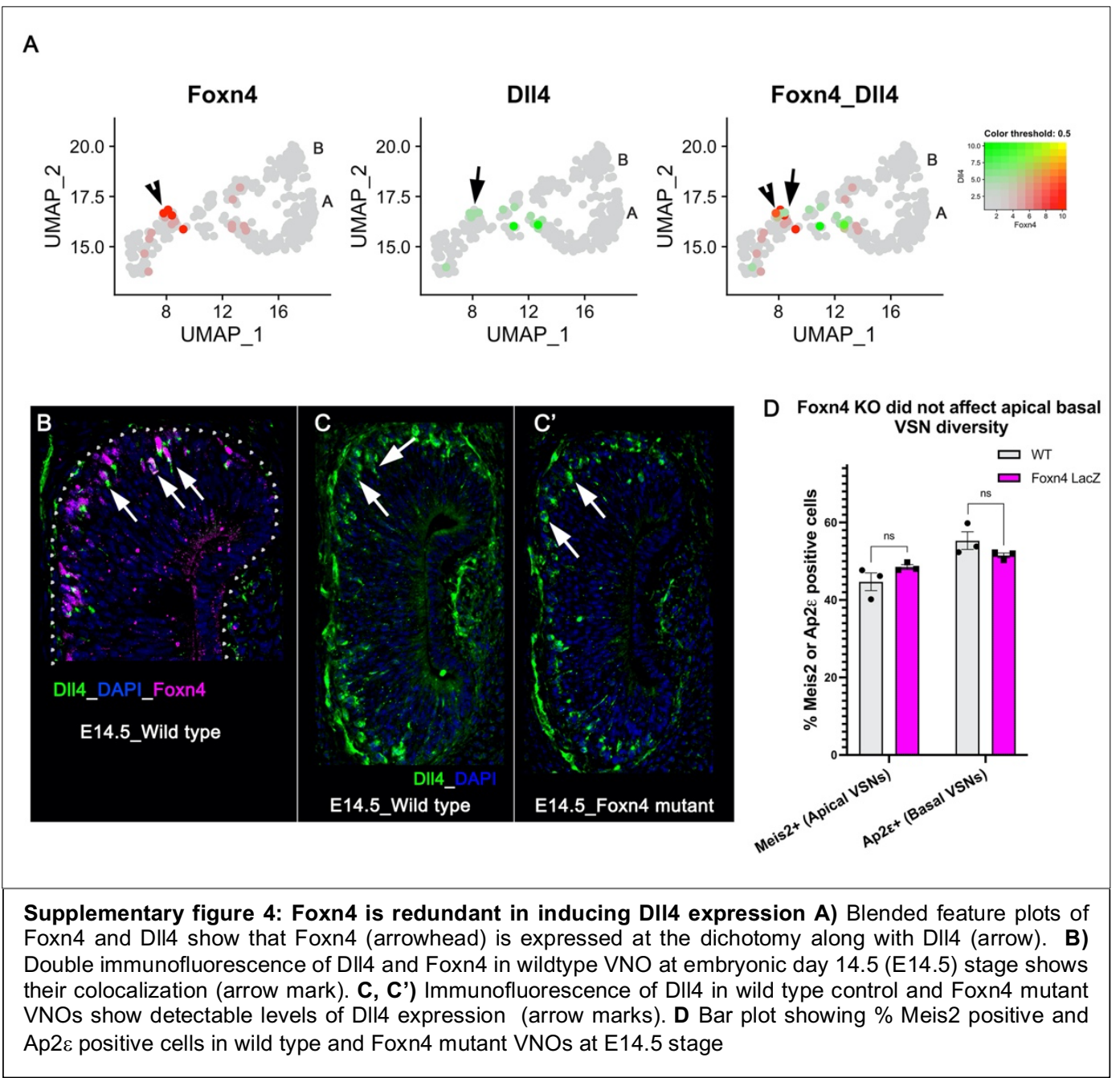


bioRxiv preprint doi: https://doi.org/10.1101/2021.10.26.466003; this version posted October 26, 2021. The copyright holder for this preprint (which was not certified by peer review) is the author/funder. All rights reserved. No reuse allowed without permission.

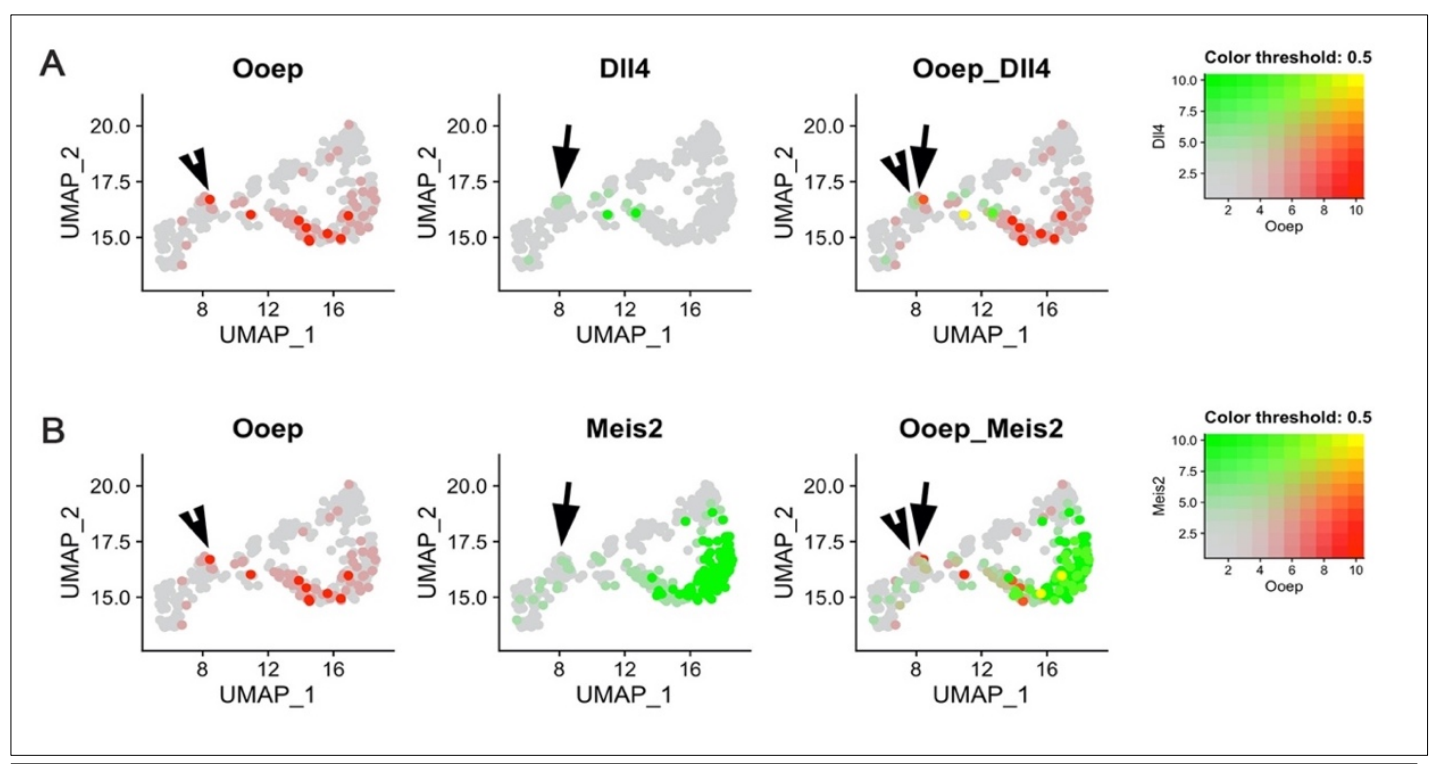

Supplementary figure5: Oocyte expression protein (Ooep) is enriched in apical specific VSNs at the dichotomy. A) Blended feature plots of Ooep and DII4 shows that Ooep (arrowhead) is initially expressed at the dichotomy, and it is enriched in DIl4 positive cells (arrow) B) Blended feature plots of Ooep and Meis2 shows that Ooep (arrowhead) is expressed along with apical VSN specific marker Meis2(arrow). 


\section{Reference:}

1. Livesey, F.J. and C.L. Cepko, Vertebrate neural cell-fate determination: lessons from the retina. Nat Rev Neurosci, 2001. 2(2): p. 109-18.

2. Bonnefont, J. and P. Vanderhaeghen, Neuronal fate acquisition and specification: time for a change. Curr Opin Neurobiol, 2021. 66: p. 195-204.

3. Louvi, A. and S. Artavanis-Tsakonas, Notch signalling in vertebrate neural development. Nat Rev Neurosci, 2006. 7(2): p. 93-102.

4. Levy, D.R., et al., The Nasopalatine Ducts Are Required for Proper Pheromone Signaling in Mice. Front Neurosci, 2020. 14: p. 585323.

5. Papes, F., D.W. Logan, and L. Stowers, The vomeronasal organ mediates interspecies defensive behaviors through detection of protein pheromone homologs. Cell, 2010. 141(4): p. 692-703.

6. Flanagan, K.A., W. Webb, and L. Stowers, Analysis of male pheromones that accelerate female reproductive organ development. PLoS One, 2011. 6(2): p. e16660.

7. He, J., et al., Encoding gender and individual information in the mouse vomeronasal organ. Science, 2008. 320(5875): p. 535-8.

8. Stowers, L., et al., Loss of sex discrimination and male-male aggression in mice deficient for TRP2. Science, 2002. 295(5559): p. 1493-500.

9. Grus, W.E., et al., Dramatic variation of the vomeronasal pheromone receptor gene repertoire among five orders of placental and marsupial mammals. Proc Natl Acad Sci U S A, 2005. 102(16): p. 5767-72.

10. Takigami, S., et al., Morphological evidence for two types of Mammalian vomeronasal system. Chem Senses, 2004. 29(4): p. 301-10.

11. Enomoto, T., et al., Bcl11b/Ctip2 controls the differentiation of vomeronasal sensory neurons in mice. J Neurosci, 2011. 31(28): p. 10159-73.

12. Young, J.M. and B.J. Trask, V2R gene families degenerated in primates, dog and cow, but expanded in opossum. Trends Genet, 2007. 23(5): p. 212-5.

13. Grus, W.E. and J. Zhang, Rapid turnover and species-specificity of vomeronasal pheromone receptor genes in mice and rats. Gene, 2004. 340(2): p. 303-12.

14. Lin, J.M., et al., The transcription factor Tfap2e/AP-2epsilon plays a pivotal role in maintaining the identity of basal vomeronasal sensory neurons. Dev Biol, 2018. 441(1): p. 67-82.

15. Katreddi, R.R. and P.E. Forni, Mechanisms underlying pre- and postnatal development of the vomeronasal organ. Cell Mol Life Sci, 2021.

16. Chamero, P., et al., G protein G(alpha)o is essential for vomeronasal function and aggressive behavior in mice. Proc Natl Acad Sci U S A, 2011. 108(31): p. 12898-903.

17. Oboti, L., et al., $A$ wide range of pheromone-stimulated sexual and reproductive behaviors in female mice depend on G protein Galphao. BMC Biol, 2014. 12: p. 31.

18. Trouillet, A.C., et al., Central role of $G$ protein Galphai2 and Galphai2(+) vomeronasal neurons in balancing territorial and infant-directed aggression of male mice. Proc Natl Acad Sci U S A, 2019. 116(11): p. 5135-5143. 
19. Palle, A., et al., Galphai2(+) vomeronasal neurons govern the initial outcome of an acute social competition. Sci Rep, 2020. 10(1): p. 894.

20. Taroc, E.Z.M., et al., Gli3 Regulates Vomeronasal Neurogenesis, Olfactory Ensheathing Cell Formation, and GnRH-1 Neuronal Migration. J Neurosci, 2020. 40(2): p. 311-326.

21. Cau, E., S. Casarosa, and F. Guillemot, Mash1 and Ngn1 control distinct steps of determination and differentiation in the olfactory sensory neuron lineage. Development, 2002. 129(8): p. 1871-80.

22. De La Rosa-Prieto, C., et al., Fate of marginal neuroblasts in the vomeronasal epithelium of adult mice. J Comp Neurol, 2009. 517(5): p. 723-36.

23. Martinez-Marcos, A., et al., Neurogenesis, migration, and apoptosis in the vomeronasal epithelium of adult mice. J Neurobiol, 2005. 63(3): p. 173-87.

24. Giacobini, P., et al., Proliferation and migration of receptor neurons in the vomeronasal organ of the adult mouse. Brain Res Dev Brain Res, 2000. 123(1): p. 33-40.

25. Stuart, T., et al., Comprehensive Integration of Single-Cell Data. Cell, 2019. 177(7): p. 1888-1902 e21.

26. Mclnnes, L., et al., UMAP: Uniform Manifold Approximation and Projection. Journal of Open Source Software, 2018. 3(29).

27. Guo, Z., et al., Expression of pax6 and sox2 in adult olfactory epithelium. J Comp Neurol, 2010. 518(21): p. 4395-418.

28. Packard, A., et al., Progenitor cell capacity of NeuroD1-expressing globose basal cells in the mouse olfactory epithelium. J Comp Neurol, 2011. 519(17): p. 3580-96.

29. Trapnell, C., et al., The dynamics and regulators of cell fate decisions are revealed by pseudotemporal ordering of single cells. Nat Biotechnol, 2014. 32(4): p. 381-386.

30. Qiu, X., et al., Reversed graph embedding resolves complex single-cell trajectories. Nat Methods, 2017. 14(10): p. 979-982.

31. Jarrett, S.M., et al., Extension of the Notch intracellular domain ankyrin repeat stack by NRARP promotes feedback inhibition of Notch signaling. Sci Signal, 2019. 12(606).

32. Ronchini, C. and A.J. Capobianco, Induction of cyclin D1 transcription and CDK2 activity by Notch(ic): implication for cell cycle disruption in transformation by Notch(ic). Mol Cell Biol, 2001. 21(17): p. 5925-34.

33. Cau, E., et al., Hes genes regulate sequential stages of neurogenesis in the olfactory epithelium. Development, 2000. 127(11): p. 2323-32.

34. Fischer, A., et al., The Notch target genes Hey1 and Hey2 are required for embryonic vascular development. Genes Dev, 2004. 18(8): p. 901-11.

35. McGill, M.A., et al., Numb regulates post-endocytic trafficking and degradation of Notch1. J Biol Chem, 2009. 284(39): p. 26427-38.

36. McGill, M.A. and C.J. McGlade, Mammalian numb proteins promote Notch1 receptor ubiquitination and degradation of the Notch1 intracellular domain. J Biol Chem, 2003. 278(25): p. 23196-203.

37. Hsu, Y.C., Theory and Practice of Lineage Tracing. Stem Cells, 2015. 33(11): p. 3197-204.

38. Kim, E.J., et al., In vivo analysis of Ascl1 defined progenitors reveals distinct developmental dynamics during adult neurogenesis and gliogenesis. J Neurosci, 2007. 27(47): p. 12764-74. 
39. Murtaugh, L.C., et al., Notch signaling controls multiple steps of pancreatic differentiation. Proc Natl Acad Sci U S A, 2003. 100(25): p. 14920-5.

40. Cheung, L., et al., NOTCH activity differentially affects alternative cell fate acquisition and maintenance. Elife, 2018. 7.

41. Taroc, E.Z.M., R.R. Katreddi, and P.E. Forni, Identifying Is/1 Genetic Lineage in the Developing Olfactory System and in GnRH-1 Neurons. Frontiers in Physiology, 2020. 11(1356).

42. Li, P., et al., Reprogramming of T cells to natural killer-like cells upon Bcl11b deletion. Science, 2010. 329(5987): p. 85-9.

43. Benedito, R. and A. Duarte, Expression of D/l4 during mouse embryogenesis suggests multiple developmental roles. Gene Expr Patterns, 2005. 5(6): p. 750-5.

44. Wakabayashi, Y. and M. Ichikawa, Distribution of Notch1-expressing cells and proliferating cells in mouse vomeronasal organ. Neurosci Lett, 2007. 411(3): p. 217-21.

45. Artavanis-Tsakonas, S., M.D. Rand, and R.J. Lake, Notch signaling: cell fate control and signal integration in development. Science, 1999. 284(5415): p. 770-6.

46. Collier, J.R., et al., Pattern formation by lateral inhibition with feedback: a mathematical model of delta-notch intercellular signalling. J Theor Biol, 1996. 183(4): p. 429-46.

47. Bray, S.J., Notch signalling in context. Nat Rev Mol Cell Biol, 2016. 17(11): p. 722-735.

48. Rodriguez, S., et al., Notch2 is required for maintaining sustentacular cell function in the adult mouse main olfactory epithelium. Dev Biol, 2008. 314(1): p. 40-58.

49. Carson, C., B. Murdoch, and A.J. Roskams, Notch 2 and Notch 1/3 segregate to neuronal and glial lineages of the developing olfactory epithelium. Dev Dyn, 2006. 235(6): p. 167888.

50. Irvin, D.K., et al., Expression patterns of Notch1, Notch2, and Notch3 suggest multiple functional roles for the Notch-DSL signaling system during brain development. J Comp Neurol, 2001. 436(2): p. 167-81.

51. Morrison, S.J., et al., Transient Notch activation initiates an irreversible switch from neurogenesis to gliogenesis by neural crest stem cells. Cell, 2000. 101(5): p. 499-510.

52. Jadhav, A.P., S.H. Cho, and C.L. Cepko, Notch activity permits retinal cells to progress through multiple progenitor states and acquire a stem cell property. Proc Natl Acad Sci U S A, 2006. 103(50): p. 18998-9003.

53. Rahe, D.P. and O. Hobert, Restriction of Cellular Plasticity of Differentiated Cells Mediated by Chromatin Modifiers, Transcription Factors and Protein Kinases. G3 (Bethesda), 2019. 9(7): p. 2287-2302.

54. Imayoshi, I., et al., Genetic visualization of notch signaling in mammalian neurogenesis. Cell Mol Life Sci, 2013. 70(12): p. 2045-57.

55. Kunnimalaiyaan, M., et al., Overexpression of the NOTCH1 intracellular domain inhibits cell proliferation and alters the neuroendocrine phenotype of medullary thyroid cancer cells. J Biol Chem, 2006. 281(52): p. 39819-30.

56. Herrick, D.B., et al., Canonical Notch Signaling Directs the Fate of Differentiating Neurocompetent Progenitors in the Mammalian Olfactory Epithelium. J Neurosci, 2018. 38(21): p. 5022-5037.

57. Herrick, D.B., et al., Notch1 maintains dormancy of olfactory horizontal basal cells, a reserve neural stem cell. Proc Natl Acad Sci U S A, 2017. 114(28): p. E5589-E5598. 
58. Fletcher, R.B., et al., Deconstructing Olfactory Stem Cell Trajectories at Single-Cell Resolution. Cell Stem Cell, 2017. 20(6): p. 817-830 e8.

59. Aydin, B., et al., Proneural factors Ascl1 and Neurog2 contribute to neuronal subtype identities by establishing distinct chromatin landscapes. Nat Neurosci, 2019. 22(6): p. 897-908.

60. Duarte, A., et al., Dosage-sensitive requirement for mouse D/l4 in artery development. Genes Dev, 2004. 18(20): p. 2474-8.

61. Koch, U., et al., Delta-like 4 is the essential, nonredundant ligand for Notch1 during thymic T cell lineage commitment. J Exp Med, 2008. 205(11): p. 2515-23.

62. Kiernan, A.E., et al., The Notch ligands DLL1 and JAG2 act synergistically to regulate hair cell development in the mammalian inner ear. Development, 2005. 132(19): p. 4353-62.

63. Del Barrio, M.G., et al., A regulatory network involving Foxn4, Mash1 and delta-like 4/Notch1 generates V2a and V2b spinal interneurons from a common progenitor pool. Development, 2007. 134(19): p. 3427-36.

64. Luo, H., et al., Forkhead box N4 (Foxn4) activates Dll4-Notch signaling to suppress photoreceptor cell fates of early retinal progenitors. Proc Natl Acad Sci U S A, 2012. 109(9): p. E553-62.

65. Misra, K., et al., Asymmetric activation of Dll4-Notch signaling by Foxn4 and proneural factors activates BMP/TGFbeta signaling to specify $V 2 b$ interneurons in the spinal cord. Development, 2014. 141(1): p. 187-98.

66. Couturier, L., K. Mazouni, and F. Schweisguth, Numb localizes at endosomes and controls the endosomal sorting of notch after asymmetric division in Drosophila. Curr Biol, 2013. 23(7): p. 588-93.

67. Bridge, G., et al., The microRNA-30 family targets DLL4 to modulate endothelial cell behavior during angiogenesis. Blood, 2012. 120(25): p. 5063-72.

68. Kavaler, J., et al., miRNA suppression of a Notch repressor directs non-neuronal fate in Drosophila mechanosensory organs. J Cell Biol, 2018. 217(2): p. 571-583.

69. Ortega, M., et al., A microRNA-mediated regulatory loop modulates NOTCH and MYC oncogenic signals in B-and T-cell malignancies. Leukemia, 2015. 29(4): p. 968-76.

70. Herr, J.C., et al., Distribution of RNA binding protein MOEP19 in the oocyte cortex and early embryo indicates pre-patterning related to blastomere polarity and trophectoderm specification. Dev Biol, 2008. 314(2): p. 300-16.

71. Li, L., B. Baibakov, and J. Dean, A subcortical maternal complex essential for preimplantation mouse embryogenesis. Dev Cell, 2008. 15(3): p. 416-425.

72. Yu, X.J., et al., The subcortical maternal complex controls symmetric division of mouse zygotes by regulating F-actin dynamics. Nat Commun, 2014. 5: p. 4887.

73. Tashiro, F., et al., Maternal-effect gene Ces5/Ooep/Moep19/Floped is essential for oocyte cytoplasmic lattice formation and embryonic development at the maternalzygotic stage transition. Genes Cells, 2010. 15(8): p. 813-28.

74. Newman, R., J. McHugh, and M. Turner, RNA binding proteins as regulators of immune cell biology. Clin Exp Immunol, 2016. 183(1): p. 37-49.

75. Bye, A.J.H., et al., The RNA-binding proteins Zfp36/1 and Zfp36/2 act redundantly in myogenesis. Skelet Muscle, 2018. 8(1): p. 37. 
76. Wheeler, J.R., et al., RNA-Binding Proteins Direct Myogenic Cell Fate Decisions. bioRxiv, 2021: p. 2021.03.14.435333.

77. Shi, P. and J. Zhang, Comparative genomic analysis identifies an evolutionary shift of vomeronasal receptor gene repertoires in the vertebrate transition from water to land. Genome Res, 2007. 17(2): p. 166-74.

78. Grus, W.E. and J. Zhang, Origin and evolution of the vertebrate vomeronasal system viewed through system-specific genes. Bioessays, 2006. 28(7): p. 709-18.

79. Takigami, S., Y. Mori, and M. Ichikawa, Projection pattern of vomeronasal neurons to the accessory olfactory bulb in goats. Chem Senses, 2000. 25(4): p. 387-93.

80. Li, S., et al., Foxn4 controls the genesis of amacrine and horizontal cells by retinal progenitors. Neuron, 2004. 43(6): p. 795-807. 